\title{
ABSOLUTE CONTINUITY OF THE LAW FOR THE TWO DIMENSIONAL STOCHASTIC NAVIER-STOKES EQUATIONS
}

\author{
BENEDETTA FERRARIO, MARGHERITA ZANELLA
}

\begin{abstract}
We consider the two dimensional Navier-Stokes equations in vorticity form with a stochastic forcing term given by a gaussian noise, white in time and coloured in space. First, we prove existence and uniqueness of a weak (in the Walsh sense) solution process $\xi$ and we show that, if the initial vorticity $\xi_{0}$ is continuous in space, then there exists a space-time continuous version of the solution. In addition we show that the solution $\xi(t, x)$ (evaluated at fixed points in time and space) is locally differentiable in the Malliavin calculus sense and that its image law is absolutely continuous with respect to the Lebesgue measure on $\mathbb{R}$.
\end{abstract}

\section{INTRODUCTION}

The analysis of stochastic partial differential equations concerns problems of existence, uniqueness ' and properties of the solution processes. In particular, there has been a lot of activity in the last years , studying the regularity in the Malliavin sense for solutions to stochastic partial differential equations. Here we are interested in looking for the existence of a density for the law of the random variable given by the solution process at fixed points in time and space. This property is important in the analysis of hitting probabilities (see [9, 11]) and concentration inequalities (see [23]). Most of the literature on this subject concerns the heat and wave equations (see e.g. [24], [1], [22], 25], [20], [19] and the ' references therein). Moreover, there is a paper dealing with the Cahn-Hilliard equation (see [5]) and 'some papers dealing with the one dimensional Burgers equation (see [21, 30]). Our aim is to deal with stochastic fluid dynamical equation in dimension bigger than one. As we shall see in Section 4 . our equation can be written as a stochastic parabolic nonlinear equation in a two dimensional spatial domain with a nonlinear term which is of a form different from that studied in other papers about stochastic parabolic nonlinear equations in spatial dimension bigger than 1 (see [20], [19]).

Therefore, we consider the two dimensional stochastic Navier-Stokes equations

$$
\begin{cases}\frac{\partial v}{\partial t}(t, x)+(v(t, x) \cdot \nabla) v(t, x)-\nu \Delta v(t, x)+\nabla p(t, x)=n(t, x) & (t, x) \in[0, T] \times D \\ \nabla \cdot v(t, x)=0 & (t, x) \in[0, T] \times D \\ v(0, x)=v_{0}(x) & x \in D .\end{cases}
$$

describing the motion of a viscous incompressible fluid in a domain $D \subset \mathbb{R}^{2}$. The unknowns are the velocity vector $v$ and the pressure $p$, whereas the data are the viscosity $\nu>0$, the initial velocity $v_{0}$ and the stochastic forcing term $n$. Suitable boundary conditions are associated to system (1.1); here we choose to work on the torus, so $D=[0,2 \pi]^{2}$ and periodic boundary conditions are assumed.

Taking formally the curl of both sides of the first equation in (1.1) we get the vorticity formulation, where the unknown is the vorticity $\xi=\nabla^{\perp} \cdot v \equiv \partial_{x_{1}} v_{2}-\partial_{x_{2}} v_{1}$. Indeed, the curl of a planar vector

2000 Mathematics Subject Classification. 60H07, 60H15, 35Q30.

Key words and phrases. Malliavin calculus, density of the solution, Gaussian noise, stochastic Navier-Stokes equations. 
filed is a vector orthogonal to the plane, hence with only one significant component $\xi$. Therefore, for regular enough solutions, system (1.1) is equivalent to

$$
\begin{cases}\frac{\partial \xi}{\partial t}(t, x)-\nu \Delta \xi(t, x)+v(t, x) \cdot \nabla \xi(t, x)=w(\mathrm{~d} x, \mathrm{~d} t) & (t, x) \in[0, T] \times D \\ \nabla \cdot v(t, x)=0 & (t, x) \in[0, T] \times D \\ \xi(t, x)=\nabla^{\perp} \cdot v(t, x) & (t, x) \in[0, T] \times D \\ \xi(0, x)=\xi_{0}(x) & x \in D\end{cases}
$$

with periodic boundary conditions. For simplicity we put $\nu=1$ from now on. The random force $w$ acting on the system is formally equal to the curl of $n$ appearing in (1.1); $w(\mathrm{~d} x, \mathrm{~d} t)$ is the formal notation for some Gaussian perturbation defined on some probability space (for the details see Subsection 2.4). We shall see that system (1.2) can be rewritten as a closed equation for the vorticity, since $v$ can be explicitly expressed in terms of $\xi$ by means of the Biot-Savart law $v=k * \xi$ (see Subsection 2.3).

We interpret Eq. (1.2) in the sense of Walsh (see [29]). Let $g(t, x, y)$ be the fundamental solution to the heat equation on the flat torus (see Subsection 2.2). We shall see that a random field $\xi=$ $\{\xi(t, x), t \in[0, T] \times D\}$ is a solution to equation (1.2) if it satisfies the evolution equation

$$
\begin{aligned}
\xi(t, x)=\int_{D} g(t, x, y) \xi_{0}(y) \mathrm{d} y+\int_{0}^{t} \int_{D} \nabla_{y} g(t-s, x, y) & \cdot v(s, y) \xi(s, y) \mathrm{d} y \mathrm{~d} s \\
& +\int_{0}^{t} \int_{D} g(t-s, x, y) w(\mathrm{~d} y, \mathrm{~d} s)
\end{aligned}
$$

with $v=k * \xi$. The stochastic integral will be explicitly defined in Subsection 2.4, Notice that in the present work we consider an additive noise, that is the stochastic forcing term is independent of the unknown process $\xi$. This particular choice is made only in order to highlight the novelties of the results when compared to the one dimensional Burgers equation. Nevertheless, with standard techniques it is possible to extend the results to the multiplicative case and this shall be the object of a subsequent paper.

In the first part of the paper we shall prove the existence and uniqueness of the solution to problem (1.2). We follow an approach similar to [15] for the one dimensional stochastic Burgers equation and to [5] for the Cahn-Hilliard stochastic equation. The regularization property of the heat kernel as stated in Lemma 6 plays a key role in our method. Since the non linear term that appears in (1.3) is non Lipschitz, we adopt a method of localization: by means of a contraction principle, we prove at first the result for the smoothed equation with truncated coefficient. This kind of result provides the uniqueness for the solution to (1.2) and its local existence, namely the existence on the time interval $[0, \tau]$ where $\tau$ is a stopping time. To prove the global existence we show that $\tau=T \mathbb{P}$-a.s. We then study the regularity of $\xi$ proving that if $\xi_{0}$ is a continuous function on $D$, then the solution admits a modification which is a space-time continuous process.

In the second part of the paper we study the regularity of the solution in the sense of stochastic calculus of variations, namely we prove the existence of the density of the random variable $\xi(t, x)$, for fixed $(t, x) \in[0, T] \times D$. For this we use the Malliavin calculus (see [24]) associated to the noise that appears in (1.2). We prove at first that for any fixed $(t, x) \in[0, T] \times D$ the random variable $\xi(t, x)$ belongs to the Sobolev space $\mathbb{D}_{\text {loc }}^{1, p}$ for every $p>4$. Then we prove that the law of $\xi(t, x)$ is absolutely continuous with respect to the Lebesgue measure on $\mathbb{R}$. We point out here that the localization argument we use in order to achieve this result does not provide the smoothness of the density since we do not have the boundedness of the derivatives of every order. Moreover, let us notice 
that the technique of analysis of the existence of the density by means of Malliavin calculus is suited for a scalar unknown; the case for a vector unknown is much more involved (see, e.g., [24]). This is the reason why we work on the Navier-Stokes equations in vorticity form (1.2) instead of the usual formulation (1.1) with respect to the vector velocity.

The main results of the paper are the following.

Theorem 1. Let $b>0$ in (2.23) and $p>2$. If $\xi_{0} \in L^{p}(D)$, then there exists a unique $\mathcal{F}_{t^{-}}$-adapted solution to equation (1.3) which is continuous with values in $L^{p}(D)$. Moreover, if $\xi_{0} \in C(D)$ the solution admits a modification which is a space-time continuous process.

Theorem 2. Let $b>1$ in (2.23). If $\xi_{0} \in C(D)$, then for every $t \in[0, T]$ and $x \in D$ the image law of the random variable $\xi(t, x)$ is absolutely continuous w.r.t. to the Lebesgue measure on $\mathbb{R}$.

The paper is organized as follows: in Section 2 we define the functional spaces, we state the results concerning the needed estimates of heat kernel on the flat torus, we present the Biot-Savart law that exploit the relation between the velocity and the vorticity and we state the hypothesis concerning the random forcing term. In Section 3 we present some technical lemmas. In Section 4 we establish the existence and uniqueness of the solution to (1.2) as well as its $\mathbb{P}$-a.s. space-time continuity. In Section 5 we prove the absolute continuity of the solution $\xi(t, x)$, for $t \in[0, T], x \in D$. Finally, the estimates of the heat kernel and its gradient are proved in $\mathrm{A}$.

Notation. In the sequel, we shall indicate with $C$ a constant that may varies from line to line. In certain cases, we write $C_{\alpha, \beta, \ldots}$ to emphasize the dependence of the constant on the parameters $\alpha, \beta, \ldots$

\section{Mathematical Setting}

2.1. Spaces and operators. We denote by $x=\left(x_{1}, x_{2}\right)$ a generic point of $\mathbb{R}^{2}$ and by

$$
x \cdot y=x_{1} y_{1}+x_{2} y_{2} \quad \text { and } \quad|x|=\sqrt{x \cdot x}, \quad x, y \in \mathbb{R}^{2}
$$

the scalar product and the norm in $\mathbb{R}^{2}$. Given $z=\mathcal{R} z+i \mathcal{I} z \in \mathbb{C}$ we denote by $|z|$ its absolute value and by $\bar{z}$ its complex coniugate: $|z|=\sqrt{(\mathcal{R} z)^{2}+(\mathcal{I} z)^{2}}, \bar{z}=\mathcal{R} z-i \mathcal{I} z$. We define $\mathbb{Z}_{+}^{2}=\left\{k=\left(k_{1}, k_{2}\right) \in\right.$ $\left.\mathbb{Z}^{2}: k_{1}>0\right\} \cup\left\{k=\left(0, k_{2}\right) \in \mathbb{Z}^{2}: k_{2}>0\right\}$ and $\mathbb{Z}_{0}^{2}=\mathbb{Z}^{2} \backslash\{0\}$.

Let $D=[0,2 \pi]^{2}$, we consider the space $L_{\sharp}^{2}(D)$ of all complex-valued $2 \pi$-periodic functions in $x_{1}$ and $x_{2}$ which are measurable and square integrable on $D$, endowed with the scalar product

$$
\langle f, g\rangle_{L^{2}(D)}=\int_{D} f(x) \overline{g(x)} \mathrm{d} x
$$

and the norm $\|\cdot\|_{L^{2}(D)}=\sqrt{\langle\cdot, \cdot\rangle_{L^{2}(D)}}$. We also consider the space $\left[L_{\sharp}^{2}(D)\right]^{2}$ consisting of all pairs $u=\left(u_{1}, u_{2}\right)$ of complex-valued periodic functions endowed with the inner product

$$
\begin{aligned}
&\langle u, v\rangle_{\left[L^{2}(D)\right]^{2}}:=\int_{D} u(x) \cdot \overline{v(x)} \mathrm{d} x \\
& \quad=\int_{D}\left[u_{1}(x) \overline{v_{1}(x)}+u_{2}(x) \overline{v_{2}(x)}\right] \mathrm{d} x, \quad u, v \in\left[L_{\sharp}^{2}(D)\right]^{2} .
\end{aligned}
$$

An orthonormal basis for the space $L_{\sharp}^{2}(D)$ is given by $\left\{e_{k}\right\}_{k \in \mathbb{Z}^{2}}$, where

$$
e_{k}(x)=\frac{1}{2 \pi} e^{i k \cdot x}, \quad x \in D, k \in \mathbb{Z}^{2} .
$$


As usual in the periodic case, we deal with mean value zero vectors. This gives a simplification in the mathematical treatment but does not prevent to consider non zero mean value vectors: this can be dealt in a similar way (see [28]). We use the notation $\dot{L}_{\sharp}^{2}(D)$ to keep tracks of the zero-mean condition. An orthonormal system for the space $\dot{L}_{\sharp}^{2}(D)$, formed by eigenfunctions of the operator $-\Delta$ with associated eigenvalues $\lambda_{k}=|k|^{2}$, is given by $\left\{e_{k}\right\}_{k \in \mathbb{Z}_{0}^{2}}$ with $e_{k}$ as in (2.1). The real-valued functions in $\dot{L}_{\sharp}^{2}(D)$ can be characterized by their Fourier series expansion as follows

$$
\dot{L}_{\sharp}^{2}(D)=\left\{f(x)=\sum_{k \in \mathbb{Z}_{0}^{2}} f_{k} e_{k}(x): \bar{f}_{k}=f_{-k} \text { for any } k, \sum_{k \in \mathbb{Z}_{0}^{2}}\left|f_{k}\right|^{2}<\infty\right\} .
$$

For every $p>2$, with $\dot{L}_{\sharp}^{p}(D)$ we denote the subspaces of $L^{p}(D)$ consisting of zero mean and periodic scalar functions. These are Banach spaces with norms inherited from $L^{p}(D)$.

Let $A$ denote the Laplacian operator $-\Delta$ with periodic boundary conditions. For every $b \in \mathbb{R}$, we define the powers of the operator $A$ as follows:

$$
\text { if } f=\sum_{k \in \mathbb{Z}_{0}^{2}} f_{k} e_{k} \quad \text { then } A^{b} f=\sum_{k \in \mathbb{Z}_{0}^{2}}|k|^{2 b} f_{k} e_{k}
$$

and

$$
D\left(A^{b}\right)=\left\{f=\sum_{k \in \mathbb{Z}_{0}^{2}} f_{k} e_{k}: \sum_{k \in \mathbb{Z}_{0}^{2}}|k|^{4 b}\left|f_{k}\right|^{2}<\infty\right\} .
$$

For any $b \in \mathbb{R}_{+}$and $p \geq 1$ we set

$$
W^{b, p}(D)=\left\{f \in \dot{L}_{\sharp}^{p}(D): A^{\frac{b}{2}} f \in \dot{L}_{\sharp}^{p}(D)\right\} .
$$

These are Banach spaces with the usual norm; when $p=2$ they become Hilbert spaces and we denote them by $W^{b}$. For $b<0$ we define $W^{b}$ as the dual space of $W^{-b}$ with respect to the $L^{2}$-scalar product.

Similarly, we proceed to define the space regularity of vector fields which are periodic, zero mean value and divergence free. We have the corresponding action of the Laplace operator on each component of the vector. Therefore we define the space

$$
H=\left\{v \in\left[\dot{L}_{\sharp}^{2}(D)\right]^{2}: \nabla \cdot v=0\right\}
$$

where the divergence free condition has to be understood in the distributional sense. This is an Hilbert space with the scalar product inherited from $\left[L^{2}(D)\right]^{2}$. We denote the norm in this space by $|\cdot|_{H}$, $|u|_{H}^{2}:=\langle u, u\rangle_{H}$. A basis for the space $H$ is $\left\{\frac{k^{\perp}}{|k|} e_{k}\right\}_{k \in \mathbb{Z}_{0}^{2}}$, where $k^{\perp}=\left(-k_{2}, k_{1}\right)$ and $e_{k}$ is given in (2.1). For $p>2$ let us set $L_{p}(D):=H \cap\left[L^{p}(D)\right]^{2}$. These are Banach spaces with norms inherited from $\left[L^{p}(D)\right]^{2}$. Similarly, for vector spaces we set

$$
H_{p}^{b}(D)=\left\{v \in L_{p}(D): A^{\frac{b}{2}} v \in L_{p}(D)\right\} .
$$

These are Banach spaces with the usual norm; when $p=2$ they become Hilbert spaces and we denote them by $H^{b}$. For $b<0$ we define $H^{b}$ as the dual space of $H^{-b}$ with respect to the $H$-scalar product.

The Poincaré inequality holds; moreover, the zero mean value assumption provides that $\|v\|_{H_{p}^{b}(D)}$ is equivalent to $\left(\|v\|_{L_{p}(D)}^{p}+\|v\|_{H_{p}^{b}(D)}^{p}\right)^{\frac{1}{p}}$.

In the sequel we shall use the Sobolev embedding Theorem (see for instance [3, Theorem 9.16]): 
- for every $2<p<\infty$ the space $H_{p}^{1}(D)$ is compactly embedded in $L_{\infty}(D)$, namely there exists a constant $C$ (depending on $p$ such that):

$$
\|v\|_{L_{\infty}(D)} \leq C\|v\|_{H_{p}^{1}(D)}
$$

- the space $W^{a}(D)$ is compactly embedded in $L^{\infty}(D)$ for $a>1$.

Notation. In the sequel, spaces over the domain $D$ will be denoted without explicitly mentioning the domain, e.g. $L^{p}$ stands for $L^{p}(D)$. By an innocuous abuse of notation, the scalar product $\langle\cdot, \cdot\rangle_{\left[L^{2}\right]^{2}}$ will be denoted by $\langle\cdot, \cdot\rangle_{L^{2}}$ and the norm $\|\cdot\|_{\left[L^{p}\right]^{2}}$ by $\|\cdot\|_{L^{p}}$.

Given two normed vector spaces $\left(U,\|\cdot\|_{U}\right)$ and $\left(V,\|\cdot\|_{V}\right)$, by $\mathcal{L}(U, V)$ we denote the space of all linear bounded operators from $U$ into $V$. We write $\langle\cdot, \cdot\rangle$ for the scalar product $\langle\cdot, \cdot\rangle_{U^{\prime} \times U}$ in the duality $U^{\prime}, U$.

2.2. The Heat Kernel. We deal with the heat kernel $g$ appearing in equation (1.3): we need suitable estimates on $g$ since its regularizing effect (see Lemma 6) will play a key role.

The operator $-A$ generates a semigroup $S(t)=e^{-t A}$ : for $\xi \in \dot{L}_{\sharp}^{2}$ and $t \in[0, T]$ we have

$$
[S(t) \xi](x)=\sum_{k \in \mathbb{Z}^{2}} e^{-|k|^{2} t}\left\langle\xi, e_{k}\right\rangle_{L^{2}} e_{k}(x)=\frac{1}{2 \pi} \sum_{k \in \mathbb{Z}^{2}}\left\langle\xi, e_{k}\right\rangle_{L^{2}} e^{-t|k|^{2}+i k \cdot x} .
$$

Moreover, the action of the semigroup on the function $\xi$ can be expressed as the convolution

$$
[S(t) \xi](x)=\int_{D} g(t, x, y) \xi(y) \mathrm{d} y
$$

where $g$ is the fundamental solution (or heat kernel) to the problem

$$
\begin{cases}\frac{\partial}{\partial t} u(t, x)-\Delta u(t, x)=0, & (t, x) \in(0, T] \times D \\ u(t, \cdot) \text { is periodic, } & t \in[0, T] \\ u(0, x)=\delta_{0}(x-y), & x, y \in D .\end{cases}
$$

By means of Fourier series expansion we recover

$$
g(t, x, y)=\frac{1}{(2 \pi)^{2}} \sum_{k \in \mathbb{Z}^{2}} e^{-t|k|^{2}+i k \cdot(x-y)} .
$$

We shall need another expression of the kernel obtained by means of the method of images (for more details see for instance [12, Chapters 2.7§5 and 2.11§3] and [26, Chapter $7 \S 2]$ ):

$$
g(t, x, y)=\frac{1}{4 \pi t} \sum_{k \in \mathbb{Z}^{2}} e^{-\frac{|x-y+2 k \pi|^{2}}{4 t}} .
$$

It is easy, using (2.6) or (2.7), to check the following properties

Proposition 3. For any $x, y \in D$ and $t>0$ we have

- Symmetry: $g(t, x, y)=g(t, y, x)$,

- $g(t, x, y)=g(t, 0, x-y)$.

Following an idea of [21], we obtain estimates on the heat kernel and its gradient in the two dimensional case.

Theorem 4. For fixed $0<s<t$ and $x \in[0,2 \pi]$ the following estimates hold: 
i. for every $0<\beta<\frac{4}{3}$ there exists a constant $C_{\beta}>0$ such that

$$
\int_{D}\left|\nabla_{y} g(s, x, y)\right|^{\beta} \mathrm{d} y \leq C_{\beta} s^{-\frac{3 \beta}{2}+1}
$$

and

$$
\int_{0}^{t} \int_{D}\left|\nabla_{y} g(s, x, y)\right|^{\beta} \mathrm{d} y \mathrm{~d} s \leq C_{\beta} t^{-\frac{3 \beta}{2}+2}
$$

ii. for every $0<\beta<2$ there exists a constant $C_{\beta}>0$ such that

$$
\int_{D}|g(s, x, y)|^{\beta} \mathrm{d} y \leq C_{\beta} s^{1-\beta}
$$

and

$$
\int_{0}^{t} \int_{D}|g(s, x, y)|^{\beta} \mathrm{d} y \mathrm{~d} s \leq C_{\beta} t^{2-\beta}
$$

This result is proven in A,

2.3. The Biot-Savart law. Now we deal with the Biot-Savart law expressing the velocity vector field $v$ in terms of the vorticity scalar field $\xi$ (we mainly refer to [17] and [18]). We have $\xi=\nabla^{\perp} \cdot v$; by taking the curl in both sides of this relationship we get

$$
\left\{\begin{array}{l}
-\Delta v=\nabla^{\perp} \xi \\
\nabla \cdot v=0 \\
v \text { periodic }
\end{array}\right.
$$

This allows to express the velocity in terms of the vorticity. In terms of Fourier series, if

$$
\xi(x)=\frac{1}{2 \pi} \sum_{k \in \mathbb{Z}_{0}^{2}} \xi_{k} e^{i k \cdot x},
$$

then

$$
v(x)=-\frac{i}{2 \pi} \sum_{k \in \mathbb{Z}_{0}^{2}} \xi_{k} \frac{k^{\perp}}{|k|^{2}} e^{i k \cdot x} .
$$

This shows that the velocity $v$ has one order more of regularity with respect to the vorticity $\xi$ : if $\xi \in W^{b-1, p}$ then $v \in H_{p}^{b}$. In particular, the norms $\|v\|_{H_{p}^{b}}$ and $\|\xi\|_{W^{b-1, p}}$ are equivalent.

In general (see, e.g., [18, Chapter 1]), the Biot-Savart law expresses the velocity in term of the vorticity as

$$
v(x)=(k * \xi)(x)=\int_{D} k(x-y) \xi(y) \mathrm{d} y,
$$

where the Biot-Savart kernel is given by

$$
k=\nabla^{\perp} G=\left(-\frac{\partial G}{\partial x_{2}}, \frac{\partial G}{\partial x_{1}}\right)
$$

and $G$ is the Green function of the Laplacian on the torus with mean zero. Notice that from (2.15) it is evident that the relation between $v$ and $\xi$ is non local in space.

We summarize the basic properties of the Biot-Savart kernel in the following lemma (see [4, Lemma $2.17])$.

Lemma 5. For every $1 \leq p<2$ the map $k$, defined above, is an $\left[L^{p}(D)\right]^{2}$ divergence-free (in the distributional sense) vector field. 
Remark 1. In principle, for every $p<2, \int_{D} k(x-y) \mathrm{d} y$ is a constant that depends on $x$, but it can be easily majored by a constant which does not depend on $x$. This is straightforward using the estimate $|\nabla G(x)| \leq C\left(|x|^{-1}+1\right)$ (see e.g. [18, Chapter 1] and [4, Proposition B.1]) and recalling that (2.16) holds.

Therefore we have some useful estimates. From (2.14), using the Sobolev embedding $H_{p}^{1} \subset L_{\infty}$ for $p>2$ and the equivalence of the norms $\|v\|_{H_{p}^{1}}$ and $\|\xi\|_{L^{p}}$ we infer that for any $p>2$ there exists a constant $C_{p}$ such that

$$
\|k * \xi\|_{L_{\infty}}=\|v\|_{L_{\infty}} \leq C_{p}\|\xi\|_{L^{p}} .
$$

From (2.15) and Lemma 5, using Young's inequality when $p \geq 1,1 \leq \alpha<2, \beta \geq 1$ with $\frac{1}{p}+1=\frac{1}{\alpha}+\frac{1}{\beta}$ we infer that

$$
\|k * \xi\|_{L_{p}}=\|v\|_{L_{p}} \leq\|k\|_{L_{\alpha}}\|\xi\|_{L^{\beta}} .
$$

2.4. The random forcing term. In this subsection we deal with the stochastic term that appears in (1.3).

Given $T>0$, let $\left(\Omega, \mathcal{F}, \mathbb{F}=\left\{\mathcal{F}_{t}\right\}_{0 \leq t \leq T}, \mathbb{P}\right)$ be a given stochastic basis. Let $Q: \dot{L}_{\sharp}^{2} \rightarrow \dot{L}_{\sharp}^{2}$ be a positive symmetric bounded linear operator. We define $L_{Q}^{2}$ as the completition of the space of all square integrable, zero mean-value, periodic functions $\varphi: D \rightarrow \mathbb{R}$ with respect to the scalar product

$$
\langle\varphi, \psi\rangle_{L_{Q}^{2}}=\langle Q \varphi, \psi\rangle_{L^{2}}
$$

Set $\mathcal{H}_{T}=L^{2}\left(0, T ; L_{Q}^{2}\right)$. This space is a real separable Hilbert space with respect to the scalar product

$$
\langle f, g\rangle_{\mathcal{H}_{T}}=\int_{0}^{T}\langle f(s), g(s)\rangle_{L_{Q}^{2}} \mathrm{~d} s=\int_{0}^{T}\langle Q f(s), g(s)\rangle_{L^{2}} \mathrm{~d} s .
$$

Let us consider the isonormal Gaussian process $W=\left\{W(h), h \in \mathcal{H}_{T}\right\}$ (see, e.g., [24]). The map $h \rightarrow W(h)$ provides a linear isometry from $\mathcal{H}_{T}$ onto $\mathcal{H}$, which is a closed subset of $L^{2}(\Omega, \mathcal{F}, \mathbb{P})$ whose elements are zero-mean Gaussian random variables. The isometry reads as

$$
\mathbb{E}(W(h) W(g))=\langle h, g\rangle_{\mathcal{H}_{T}} .
$$

We understand the stochastic term appearing in equation (1.3) in the following sense: for $h \in \mathcal{H}_{T}$, we set

$$
W(h)=\int_{0}^{T} \int_{D} h(s, y) w(\mathrm{~d} y, \mathrm{~d} s)
$$

namely, $\int_{0}^{T} \int_{D} h(s, y) w(\mathrm{~d} y, \mathrm{~d} s)$ is a zero-mean Gaussian random variable with covariance $\mathbb{E}\left[W(h)^{2}\right]=$ $\|h\|_{\mathcal{H}_{T}}^{2}$.

We point out that the stochastic term introduced above by means of the linear isometry $W$ can be understood in the setting introduced by Da Prato-Zabczyk in [8] as well as in the setting introduced by Walsh in [29]. First, we can write $W(h)$ as

$$
W(h)=\sum_{j} \int_{0}^{T}\left\langle h(s, \cdot), \tilde{e}_{j}\right\rangle_{L_{Q}^{2}} \mathrm{~d} \beta_{s}\left(\tilde{e}_{j}\right)
$$

where $\left\{\tilde{e}_{j}\right\}_{j}$ is a complete orthonormal basis of $L_{Q}^{2}$ and $\beta_{s}\left(\tilde{e}_{j}\right)=W\left(1_{[0, s]} \tilde{e}_{j}\right)$; hence $\left\{\beta\left(\tilde{e}_{j}\right)\right\}_{j}$ is a sequence of independent standard one-dimensional Brownian motions on $(\Omega, \mathcal{F}, \mathbb{P})$ adapted to $\left\{\mathcal{F}_{t}\right\}_{t \in[0, T]}$. By setting $M_{t}(A):=W\left(\mathbf{1}_{[0, t]} \mathbf{1}_{A}\right)$ for all $t \in[0, T]$ and $A \in \mathcal{B}_{b}\left(\mathbb{R}^{2}\right)$, we construct a martingale measure with covariance $Q$ and (see e.g. [10]) (2.21) coincides with the stochastic integral in the Walsh sense. 
Moreover, the isonormal Gaussian process $W$ can be associated to a $Q$-Wiener process $\mathcal{W}_{t}$ on $\dot{L}_{\sharp}^{2}$ (as defined in [8]) in the following way:

$$
\left\langle\mathcal{W}_{t}, h\right\rangle_{L^{2}}=W\left(\mathbf{1}_{[0, t]} h\right) \quad \forall h \in \dot{L}_{\sharp}^{2},
$$

and (2.20) coincides with the integral w.r.t. $\mathcal{W}$, in a sense made precise in [10, Section 3.4]. The stochastic convolution appearing in (1.3) has now to be understood in the described ways. Notice that, by construction, the random forcing term is periodic and with zero mean in the space variable. Since we are in a spatial domain of dimension larger than one, it is not surprising (see, e.g., [8]) that we cannot consider $Q$ to be the indentity, but we need $Q$ to have some regularizing effect. We choose to work with a covariance operator of the form

$$
Q=(-\Delta)^{-b}
$$

for some $b>0$. This means that

$$
Q e_{k}=|k|^{-2 b} e_{k} \quad \forall k \in \mathbb{Z}_{0}^{2}
$$

and a complete orthonormal basis of $L_{Q}^{2}$ is given by $\tilde{e}_{k}(x)=\frac{1}{\sqrt{2} \pi}|k|^{b} \cos (k \cdot x)$ and $\tilde{e}_{-k}(x)=\frac{1}{\sqrt{2} \pi}|k|^{b} \sin (k$. $x)$ for $k \in \mathbb{Z}_{+}^{2}$. Notice that the choice of $Q$ as in (2.23) is made only in order to simplify some computations but it does not prevent to consider a more general operator $Q$ which does not commute with the Laplacian operator or which has finite dimensional range. By $\operatorname{Tr} Q$ we denote the trace of the operator $Q$. If $Q$ is as in (2.23) then $\operatorname{Tr} Q=\sum_{k \in \mathbb{Z}_{0}^{2}}|k|^{-2 b}$.

Let us show that when $b>0$ in (2.23) the stochastic integral $\int_{0}^{t} \int_{D} g(t-s, x, y) w(\mathrm{~d} y, \mathrm{~d} s)$ is well defined. This is equivalent to have $g(t-\cdot, x, \cdot) \in \mathcal{H}_{t}$ for every $t>0$. Indeed,

$$
\begin{aligned}
\|g(t-\cdot, x, \cdot)\|_{\mathcal{H}_{t}}^{2} & =\int_{0}^{t}\|g(t-s, x, \cdot)\|_{L_{Q}^{2}}^{2} \mathrm{~d} s=\int_{0}^{t}\langle Q g(t-s, x, \cdot), g(t-s, x, \cdot)\rangle_{L^{2}} \mathrm{~d} s \\
& =\int_{0}^{t}\left\|Q^{\frac{1}{2}} g(t-s, x, \cdot)\right\|_{L^{2}}^{2} \mathrm{~d} s=\int_{0}^{t} \sum_{k \in \mathbb{Z}_{0}^{2}}\left|\left\langle e_{k}, Q^{\frac{1}{2}} g(t-s, x, \cdot)\right\rangle_{L^{2}}\right|^{2} \mathrm{~d} s \\
& =\sum_{k \in \mathbb{Z}_{0}^{2}} \int_{0}^{t}\left|\left\langle Q^{\frac{1}{2}} e_{k}, g(t-s, x, \cdot)\right\rangle_{L^{2}}\right|^{2} \mathrm{~d} s \\
& =\sum_{k \in \mathbb{Z}_{0}^{2}}|k|^{-2 b} \int_{0}^{t}\left|\left\langle e_{k}, g(t-s, x, \cdot)\right\rangle_{L^{2}}\right|^{2} \mathrm{~d} s \\
& =\sum_{k \in \mathbb{Z}_{0}^{2}}|k|^{-2 b} \int_{0}^{t} e^{-2|k|^{2}(t-s)}\left|e_{k}(x)\right|^{2} \mathrm{~d} s \quad \text { by (2.6) } \\
& =\frac{1}{(2 \pi)^{2}} \sum_{k \in \mathbb{Z}_{0}^{2}}|k|^{-2 b} \frac{\left(1-e^{-2|k|^{2} t}\right)}{2|k|^{2}} \quad \text { since }\left|e_{k}(x)\right|=\frac{1}{2 \pi} \\
& \leq \frac{1}{2(2 \pi)^{2}} \sum_{k \in \mathbb{Z}_{0}^{2}}|k|^{-2-2 b .}
\end{aligned}
$$

The latter series is convergent if and only if $b>0$. The hypothesis $b>0$ is sufficient for the space-time continuity of the stochastic convolution's trajectories as well (see [6, Theorem 2.13]). 
Remark 2. Notice that, since we work on the flat torus, we have good estimates on the norm of the normalized eigenfunctions $e_{k}$ of the Laplacian. Thanks to this fact we have rather weak assumptions on the covariance operator of the noise, i.e. the exponent $b$ in (2.23). However, in a general domain of $\mathbb{R}^{2}$ with smooth boundary, the growth of normalized eigenfunctions is more difficult to control. Useful estimates for this case are provided for instance in [13].

\section{Some PRELIMinARIES LEMmas}

In this Section we establish some estimates showing the regularizing effect of convolution with the gradient of the kernel $g$ or with $g$ itself, as they appear in the formulation (1.3) à la Walsh of our problem.

Let $J$ be the linear operator defined as

$$
(J \varphi)(t, x):=\int_{0}^{t} \int_{D} \nabla_{y} g(t-s, x, y) \cdot \varphi(s, y) \mathrm{d} y \mathrm{~d} s,
$$

for $t \in[0, T], x \in D$. We have that $J$ is well defined in some spaces as defined in the following lemma.

Lemma 6. i) Let $p \geq 1, \alpha \geq 1,1 \leq \beta<\frac{4}{3}, \gamma>\frac{2 \beta}{2-\beta}$ such that $\frac{1}{\beta}=1+\frac{1}{p}-\frac{1}{\alpha}$.

Then $J$ is a bounded linear operator from $L^{\gamma}\left(0, T ; L_{\alpha}\right)$ into $L^{\infty}\left(0, T ; L^{p}\right)$. Moreover there exists a constant $C_{\beta}$ such that

$$
\begin{gathered}
\|J(\varphi)(t, \cdot)\|_{L^{p}} \leq C_{\beta} \int_{0}^{t}(t-s)^{\frac{1}{\beta}-\frac{3}{2}}\|\varphi(s, \cdot)\|_{L_{\alpha}} \mathrm{d} s \\
\|J(\varphi)(t, \cdot)\|_{L^{p}} \leq C_{\beta} t^{\frac{1}{\beta}-\frac{3}{2}+\frac{\gamma-1}{\gamma}}\left(\int_{0}^{t}\|\varphi(s, \cdot)\|_{L_{\alpha}}^{\gamma} \mathrm{d} s\right)^{\frac{1}{\gamma}}
\end{gathered}
$$

for all $t \in[0, T]$.

ii) Let $p>4$ and $\gamma>\frac{2 p}{p-2}$. Then the operator $J$ maps $L^{\gamma}\left(0, T ; L_{p}\right)$ into $C([0, T] \times D)$. Moreover there exists a constant $C_{T, p}$ such that

$$
\sup _{0 \leq t \leq T} \sup _{x \in D}|(J \varphi)(t, x)| \leq C_{T, p}\left(\int_{0}^{T}\|\varphi(r, \cdot)\|_{L_{p}}^{\gamma} \mathrm{d} r\right)^{\frac{1}{\gamma}} .
$$

Proof. These results are inspired by [14, Lemma 3.1], but we need to perform all the computations since now we are in a two dimensional domain.

We first prove i). Using the continuous version of Minkowski's inequality (see e.g. [27, Theorem 6.2.14]), then Young's inequality with $\frac{1}{\alpha}+\frac{1}{\beta}=1+\frac{1}{p}$, and finally Hölder's inequality with $\gamma>\frac{2 \beta}{2-\beta}$ we get

$$
\begin{aligned}
& \left\|\int_{0}^{t} \int_{D} \nabla_{y} g(t-s, \cdot, y) \cdot \varphi(s, y) \mathrm{d} y \mathrm{~d} s\right\|_{L^{p}} \\
& \leq \int_{0}^{t}\left\|\int_{D} \nabla_{y} g(t-s, \cdot, y) \cdot \varphi(s, y) \mathrm{d} y\right\|_{L^{p}} \mathrm{~d} s=\int_{0}^{t}\left\|\nabla_{y} g(t-s, 0, \cdot) * \varphi(s, \cdot)\right\|_{L^{p}} \mathrm{~d} s \\
& \quad \leq \int_{0}^{t}\left\|\nabla_{y} g(t-s, 0, \cdot)\right\|_{L^{\beta}}\|\varphi(s, \cdot)\|_{L_{\alpha}} \mathrm{d} s \leq C_{\beta} \int_{0}^{t}(t-s)^{\frac{1}{\beta}-\frac{3}{2}}\|\varphi(s, \cdot)\|_{L_{\alpha}} \mathrm{d} s \quad \text { by (2.8). }
\end{aligned}
$$


This proves (3.2). By Hölder's inequality we estimate the latter quantity by

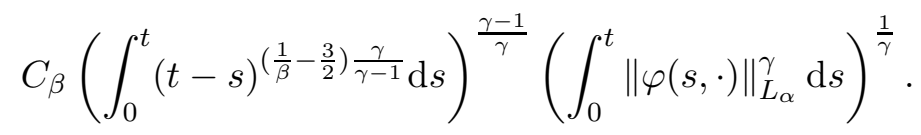

Calculating the first time integral we obtain (3.3).

As regards ii), we use the factorization method (for more details see, e.g., [6, Section 2.2.1]), which is based on the equality

$$
\frac{\pi}{\sin (\pi a)}=\int_{s}^{t}(t-r)^{a-1}(r-s)^{-a} \mathrm{~d} r, \quad a \in(0,1)
$$

We also use the Chapman-Kolmogorov relation for $s<r<t$

$$
\int_{D} g(t-r, x, z) g(r-s, z, y) \mathrm{d} z=g(t-s, x, y)
$$

which, thanks to the symmetry of the kernel $g$ in the space variables, gives

$$
\begin{aligned}
\int_{D} \partial_{z_{i}} g(t-r, x & , z) g(r-s, z, y) \mathrm{d} z=\int_{D}-\partial_{x_{i}} g(t-r, x, z) g(r-s, z, y) \mathrm{d} z \\
& =-\partial_{x_{i}} \int_{D} g(t-r, x, z) g(r-s, z, y) \mathrm{d} z=-\partial_{x_{i}} g(t-s, x, y)=\partial_{y_{i}} g(t-s, x, y)
\end{aligned}
$$

Let us show that $J \varphi$, defined in (3.1), has an equivalent expression given by

$$
(J \varphi)(t, x)=\frac{\sin (\pi a)}{\pi} \int_{0}^{t}(t-r)^{a-1}\left(\int_{D} \nabla_{z} g(t-r, x, z) \cdot Y^{a}(r, z) \mathrm{d} z\right) \mathrm{d} r
$$

with

$$
Y^{a}(r, z)=\int_{0}^{r} \int_{D}(r-s)^{-a} g(r-s, z, y) \varphi(s, y) \mathrm{d} y \mathrm{~d} s
$$

For this it is enough to check that

$$
\begin{aligned}
\int_{0}^{t} \int_{D} \partial_{y_{i}} g(t-s, x, y) \varphi_{i}(s, y) \mathrm{d} y \mathrm{~d} s & \\
& =\frac{\sin (\pi a)}{\pi} \int_{0}^{t}(t-r)^{a-1}\left(\int_{D} \partial_{z_{i}} g(t-r, x, z) Y_{i}^{a}(r, z) \mathrm{d} z\right) \mathrm{d} r
\end{aligned}
$$


for $i=1,2$. Let us work on the r.h.s.; keeping in mind the definition of $Y_{i}^{a}$ and by means of Fubini theorem we infer that

$$
\begin{aligned}
\int_{0}^{t}(t-r)^{a-1}\left(\int_{D} \partial_{z_{i}} g(t-r, x, z) Y_{i}^{a}(r, z) \mathrm{d} z\right) \mathrm{d} r & \int_{0}^{t}(t-r)^{a-1}\left(\int_{D} \partial_{z_{i}} g(t-r, x, z)\right. \\
& \left.\quad\left[\int_{0}^{r} \int_{D}(r-s)^{-a} g(r-s, z, y) \varphi_{i}(s, y) \mathrm{d} y \mathrm{~d} s\right] \mathrm{d} z\right) \mathrm{d} r \\
= & \int_{0}^{t}\left(\int_{s}^{t}(t-r)^{a-1}(r-s)^{-a}\right. \\
& {\left.\left[\int_{D}\left[\int_{D} \partial_{z_{i}} g(t-r, x, z) g(r-s, z, y) \mathrm{d} z\right] \varphi_{i}(s, y) \mathrm{d} y\right] \mathrm{d} r\right) \mathrm{d} s } \\
= & \int_{0}^{t}\left(\int_{s}^{t}(t-r)^{a-1}(r-s)^{-a}\left[\int_{D} \partial_{y_{i}} g(t-s, x, y) \varphi_{i}(s, y) \mathrm{d} y\right] \mathrm{d} r\right) \mathrm{d} s \quad \text { by (3.6) } \\
= & \frac{\pi}{\sin (\pi a)} \int_{0}^{t} \int_{D} \partial_{y_{i}} g(t-s, x, y) \varphi_{i}(s, y) \mathrm{d} y \mathrm{~d} s \quad \text { by (3.5) } .
\end{aligned}
$$

This proves (3.7). Therefore, by Hölder's inequality we get

$$
\begin{aligned}
|(J \varphi)(t, x)| & \leq \frac{\sin (\pi a)}{\pi} \int_{0}^{t}(t-r)^{a-1}\left\|\nabla_{z} g(t-r, x, \cdot)\right\|_{L^{\frac{p}{p-1}}}\left\|Y^{a}(r, \cdot)\right\|_{L_{p}} \mathrm{~d} r \\
& \leq C_{p} \frac{\sin (\pi a)}{\pi} \int_{0}^{t}(t-r)^{a-1-\frac{3}{2}+\frac{p-1}{p}}\left\|Y^{a}(r, \cdot)\right\|_{L_{p}} \mathrm{~d} r \text { by (2.8) if } p>4 .
\end{aligned}
$$

Now we estimate $\left\|Y^{a}(r, \cdot)\right\|_{L_{p}}$; by means of Minkowsky's and Young's inequalities and using (2.11) we infer that

$$
\begin{aligned}
\left\|Y^{a}(r, \cdot)\right\|_{L_{p}} & =\left\|\int_{0}^{r} \int_{D}(r-s)^{-a} g(r-s, \cdot, y) \varphi(s, y) \mathrm{d} y \mathrm{~d} s\right\|_{L_{p}} \\
& \leq \int_{0}^{r}(r-s)^{-a}\left\|\int_{D} g(r-s, \cdot, y) \varphi(s, y) \mathrm{d} y\right\|_{L_{p}} \mathrm{~d} s \\
& =\int_{0}^{r}(r-s)^{-a}\|g(r-s, 0, \cdot) * \varphi(s, \cdot)\|_{L_{p}} \mathrm{~d} s \\
& \leq \int_{0}^{r}(r-s)^{-a}\|g(r-s, 0, \cdot)\|_{L_{1}}\|\varphi(s, \cdot)\|_{L_{p}} \mathrm{~d} s \\
& \leq C \int_{0}^{r}(r-s)^{-a}\|\varphi(s, \cdot)\|_{L_{p}} \mathrm{~d} s .
\end{aligned}
$$

Collecting the above estimates, by means of Fubini theorem we obtain that

$$
\begin{aligned}
|(J \varphi)(t, x)| & \leq C \frac{\sin (\pi a)}{\pi} \int_{0}^{t}(t-r)^{a-\frac{3}{2}-\frac{1}{p}}\left(\int_{0}^{r}(r-s)^{-a}\|\varphi(s, \cdot)\|_{L_{p}} \mathrm{~d} s\right) \mathrm{d} r \\
& =C \frac{\sin (\pi a)}{\pi} \int_{0}^{t}\|\varphi(s, \cdot)\|_{L^{p}}\left(\int_{s}^{t}(t-r)^{a-\frac{3}{2}-\frac{1}{p}}(r-s)^{-a} \mathrm{~d} r\right) \mathrm{d} s .
\end{aligned}
$$


With the change of variables $r=s+z(t-s)$ we can compute the inner integral as follows:

$$
\begin{aligned}
\int_{s}^{t}(t-r)^{a-\frac{3}{2}-\frac{1}{p}}(r-s)^{-a} \mathrm{~d} r & =(t-s)^{-\frac{p+2}{2 p}} \int_{0}^{1}(1-z)^{a-\frac{3}{2}-\frac{1}{p}} z^{-a} d z \\
& =(t-s)^{-\frac{p+2}{2 p}} \int_{0}^{1}(1-z)^{a-1-\frac{p+2}{2 p}} z^{-a} d z
\end{aligned}
$$

The latter integral is equal to the beta function $B\left(1-a, a-\frac{p+2}{2 p}\right)$, which is finite provided $\frac{p+2}{2 p}<$ $a<1$; therefore given $p>4$ we choose $a \in\left(\frac{p+2}{2 p}, 1\right)$. Hence

$$
\begin{aligned}
|(J \varphi)(t, x)| & \leq C_{p} \int_{0}^{t}(t-s)^{-\frac{p+2}{2 p}}\|\varphi(s, \cdot)\|_{L_{p}} \mathrm{~d} s \\
& \leq C_{p}\left(\int_{0}^{t}(t-s)^{-\frac{p+2}{2 p} \frac{\gamma}{\gamma-1}} \mathrm{~d} s\right)^{\frac{\gamma-1}{\gamma}}\left(\int_{0}^{t}\|\varphi(s, \cdot)\|_{L_{p}}^{\gamma} \mathrm{d} s\right)^{\frac{1}{\gamma}} \\
& \leq C_{T, p}\left(\int_{0}^{T}\|\varphi(s, \cdot)\|_{L_{p}}^{\gamma} \mathrm{d} s\right)^{\frac{1}{\gamma}}
\end{aligned}
$$

for $\frac{p+2}{2 p} \frac{\gamma}{\gamma-1}<1$, i.e. $\gamma>\frac{2 p}{p-2}$.

The above estimate shows that $J \varphi \in L^{\infty}([0, T] \times D)$ for every $\varphi \in L^{\gamma}\left(0, T ; L_{p}\right)$. It remains to prove that $J \varphi \in C([0, T] \times D)$. Let us notice that for step functions $\varphi, J \varphi$ is a space-time continuous function; this follows from the well posedness of the integral $\int_{0}^{t} \int_{D} \nabla_{y} g(t-s, x, y) \mathrm{d} y \mathrm{~d} s$ (let us recall that $\int_{0}^{t} \int_{D}\left|\nabla_{y} g(t-s, x, y)\right| \mathrm{d} y \mathrm{~d} s<\infty$, see (2.9) $)$. This kind of regularity can be then extended to every $\varphi \in L^{\gamma}\left(0, T ; L_{p}\right)$ by a standard approximation procedure.

The second result concerns the stochastic integral in equation (1.3), i.e. the process

$$
z(t, x)=\int_{0}^{t} \int_{D} g(t-s, x, y) w(\mathrm{~d} y, \mathrm{~d} s)
$$

solution of

$$
\left\{\begin{array}{l}
\frac{\partial z}{\partial t}(t, x)-\Delta z(t, x)=w(\mathrm{~d} x, \mathrm{~d} t) \\
z(0, x)=0
\end{array}\right.
$$

We have

Lemma 7. Let $b>0$ in (2.23) and $p>2$. Then

$$
\mathbb{E}\left[\sup _{t \in[0, T]}\|z(t, \cdot)\|_{L^{p}}^{p}\right]<\infty .
$$

Moreover, $\mathbb{P}$-a.s. $z$ is a continuous function on $[0, T] \times D$.

Proof. We use the factorization method. Given $\alpha \in\left(0, \frac{1}{2}\right)$ we can represent $z$ as

$$
z(t, x)=\frac{\sin (\pi \alpha)}{\pi} \int_{0}^{t}(t-\sigma)^{\alpha-1}\left(\int_{D} g(t-\sigma, x, z) Z^{\alpha}(\sigma, z) \mathrm{d} z\right) \mathrm{d} \sigma
$$


with

$$
Z^{\alpha}(\sigma, z):=\int_{0}^{\sigma}(\sigma-s)^{-\alpha} g(\sigma-s, z, y) w(\mathrm{~d} y, \mathrm{~d} s)
$$

From (2.24) we know that $Z^{\alpha}(\sigma, z)$ is a zero-mean real gaussian random variable with covariance given by

$$
\begin{aligned}
\mathbb{E}\left|Z^{\alpha}(\sigma, z)\right|^{2} & =\sum_{k \in \mathbb{Z}_{0}^{2}}|k|^{-2 b} \int_{0}^{\sigma} e^{-2|k|^{2}(\sigma-s)}(\sigma-s)^{-2 \alpha}\left|e_{k}(z)\right|^{2} \mathrm{~d} s \\
& \leq \frac{2^{2 \alpha-1}}{(2 \pi)^{2}} \Gamma(1-2 \alpha) \sum_{k \in \mathbb{Z}_{0}^{2}}|k|^{2(2 \alpha-1-b)}
\end{aligned}
$$

where the Gamma function is finite provided $\alpha \in\left(0, \frac{1}{2}\right)$. The latter series converges if and only if $b>2 \alpha$. Therefore, from the gaussianity of $Z^{\alpha}$, there exists $C_{p}>0$ such that

$$
\mathbb{E}\left|Z^{\alpha}(\sigma, z)\right|^{p} \leq C_{p}\left(\mathbb{E}\left|Z^{\alpha}(\sigma, z)\right|^{2}\right)^{\frac{p}{2}}<\infty
$$

and we have

$$
\int_{0}^{T} \mathbb{E}\left\|Z^{\alpha}(\sigma, \cdot)\right\|_{L^{p}}^{p} \mathrm{~d} \sigma=\int_{0}^{T} \int_{D} \mathbb{E}\left|Z^{\alpha}(\sigma, z)\right|^{p} \mathrm{~d} z \mathrm{~d} \sigma<\infty .
$$

From Minkowsky's, Young's and Hölder's inequalities we infer that

$$
\begin{aligned}
\|z(t, \cdot)\|_{L^{p}} & \leq \frac{\sin (\pi \alpha)}{\pi} \int_{0}^{t}(t-\sigma)^{\alpha-1}\left\|\int_{D} g(t-\sigma, \cdot, z) Z^{\alpha}(\sigma, z) \mathrm{d} z\right\|_{L^{p}} \mathrm{~d} \sigma \\
& \leq \frac{\sin (\pi \alpha)}{\pi} \int_{0}^{t}(t-\sigma)^{\alpha-1}\|g(t-\sigma, 0, \cdot)\|_{L^{1}}\left\|Z^{\alpha}(\sigma, \cdot)\right\|_{L^{p}} \mathrm{~d} \sigma \\
& \leq \frac{\sin (\pi \alpha)}{\pi} \int_{0}^{t}(t-\sigma)^{\alpha-1}\left\|Z^{\alpha}(\sigma, \cdot)\right\|_{L^{p}} \mathrm{~d} \sigma \quad \text { by (2.10) } \\
& \leq C_{T, \alpha}\left(\int_{0}^{t}\left\|Z^{\alpha}(\sigma, \cdot)\right\|_{L^{p}}^{p} \mathrm{~d} \sigma\right)^{\frac{1}{p}}
\end{aligned}
$$

provided $p>\frac{1}{\alpha}$. Then

$$
\begin{aligned}
\mathbb{E}\left[\sup _{t \in[0, T]}\|z(t, \cdot)\|_{L^{p}}^{p}\right] & \leq C_{T, \alpha} \mathbb{E}\left[\sup _{t \in[0, T]} \int_{0}^{t}\left\|Z^{\alpha}(\sigma, \cdot)\right\|_{L^{p}}^{p} \mathrm{~d} \sigma\right] \\
& =C_{T, \alpha} \int_{0}^{T} \mathbb{E}\left\|Z^{\alpha}(\sigma, \cdot)\right\|_{L^{p}}^{p} \mathrm{~d} \sigma<\infty
\end{aligned}
$$

for any $p>2$. This proves (3.10).

As regards the proof of the existence of a space-time continuous modification of $z$ it is similar to 6 , Theorem 2.13] and it follows from [6, Lemma 2.12]. In fact, by the semigroup representation of the heat kernel we can write

$$
z(t, x)=\frac{\sin (\pi \alpha)}{\pi} \int_{0}^{t}(t-\sigma)^{\alpha-1}\left[S(t-\sigma) Z^{\alpha}(\sigma, \cdot)\right](x) \mathrm{d} \sigma, \quad x \in D, t \in[0, T] .
$$

Since we are dealing with the heat kernel on a flat torus and we are working under the assumption $b>0$, we are in the framework given by [6, Hypothesis 2.10]. Then it is sufficient to prove that $Z^{\alpha} \in L^{2 m}([0, T] \times D)$ for $m>\frac{1}{\alpha}$. This immediately follows from (3.11). 


\section{Existence AND UniqUeness of The SOlution}

The main aim of this Section is to prove the existence and uniqueness of the solution to the SPDE (1.2) as stated in Theorem 1. Since the derivative of $w$ is formal, we consider the equation in a weak sense, as in [29] for the stochastic heat equation. In order to simplify the notation, recalling the relation between the vorticity scalar field $\xi$ and velocity vector field $v$ given by the Biot-Savart law (2.15), let us define the vector field $q(\xi)=\xi(k * \xi)$, i.e.

$$
[q(\xi)](x)=\xi(x) \int_{D} k(x-y) \xi(y) \mathrm{d} y .
$$

By means of Hölder's inequality, from (2.17) if $p>2$ we know that

$$
\|q(\xi)\|_{L_{p}} \leq\|\xi\|_{L^{p}}\|k * \xi\|_{L_{\infty}} \leq C_{p}\|\xi\|_{L^{p}}^{2}
$$

namely $q: L^{p} \rightarrow L_{p}$ for any $p>2$. This allows to write system (1.2) in an equivalent form, where the velocity does not appear anymore.

Since $v=k * \xi$ is divergence free, for the nonlinear term in equation (1.2) we have

$$
v \cdot \nabla \xi=\nabla \cdot(v \xi)=\nabla \cdot q(\xi) .
$$

Therefore we give this definition of solution to system (1.2). This is a weak solution in the sense of PDE's, hence involving test functions $\varphi$.

Definition 8. We say that an $\dot{L}_{\sharp}^{2}$-valued continuous $\mathcal{F}_{t}$-adapted stochastic process $\xi$ is a solution to (1.2) if it solves (1.2) in the following sense: for every $t \in[0, T], \varphi \in W^{a}$ with a $>2$ we have

$$
\begin{aligned}
\int_{D} \xi(t, x) \varphi(x) \mathrm{d} x-\int_{0}^{t} \int_{D} \xi(s, x) \Delta \varphi(x) \mathrm{d} x \mathrm{~d} s & -\int_{0}^{t} \int_{D} q(\xi(s, \cdot))(x) \cdot \nabla \varphi(x) \mathrm{d} x \mathrm{~d} s \\
& =\int_{D} \xi_{0}(x) \varphi(x) \mathrm{d} x+\int_{0}^{t} \int_{D} \varphi(x) w(\mathrm{~d} x, \mathrm{~d} s)
\end{aligned}
$$

$\mathbb{P}$-a.s.

Notice that the non linear term is well defined since, using repeatedly Hölder's inequality and the Sobolev embedding, we obtain

$$
\begin{aligned}
\left|\int_{D} q(\xi(s, \cdot))(x) \cdot \nabla \varphi(x) \mathrm{d} x\right| & \leq\|\nabla \varphi\|_{L^{\infty}}\|q(\xi(s, \cdot))\|_{L_{1}} \\
& \leq C\|\nabla \varphi\|_{W^{s}}\|k * \xi(s, \cdot)\|_{L_{2}}\|\xi(s, \cdot)\|_{L^{2}} \quad \text { if } s>1 \\
& \leq C\|\varphi\|_{W^{s+1}}\|\xi(s, \cdot)\|_{L^{2}}^{2} \text { by (2.18) }(\alpha=1, \beta=p=2) .
\end{aligned}
$$

Following the idea of [29] for the heat equation or of [14] for the Burgers equation one obtains that this is equivalent to ask that for any $(t, x) \in[0, T] \times D$

$$
\begin{aligned}
\xi(t, x)=\int_{D} g(t, x, y) \xi_{0}(y) \mathrm{d} y+\int_{0}^{t} \int_{D} \nabla_{y} g(t-s, x, y) \cdot q(\xi(s, \cdot))(y) \mathrm{d} y \mathrm{~d} s & \\
& +\int_{0}^{t} \int_{D} g(t-s, x, y) w(\mathrm{~d} y, \mathrm{~d} s)
\end{aligned}
$$

P-a.s.

The non linear term $q(\xi)$ that appears in (4.4) is non Lipschitz. Therefore, we use a localization argument to prove the existence and uniqueness of the solution. By means of a fixed point argument 
we prove at first the existence and uniqueness result for a local solution; then the global result follows from suitable estimates on the process $\xi$.

So, we first solve the problem when the nonlinearity is truncated to be globally Lipschitz.

4.1. The case of truncated nonlinearity. Let $N \geq 1$ and denote by $\Theta_{N}:[0,+\infty) \rightarrow[0,1]$ a $C^{1}$ function such that $\left|\Theta_{N}^{\prime}(s)\right| \leq 2$ for any $s \geq 0$ and

$$
\Theta_{N}(s)= \begin{cases}1 & \text { if } 0 \leq s<N \\ 0 & \text { if } s \geq N+1\end{cases}
$$

Given $\xi \in L^{p}$, for $p>2$, we define

$$
\begin{aligned}
& q_{N}(\xi)=q(\xi) \Theta_{N}\left(\|\xi\|_{L^{p}}\right), \\
& \tilde{q}_{N}(\xi)=q(\xi) \Theta_{N}^{\prime}\left(\|\xi\|_{L^{p}}\right) .
\end{aligned}
$$

By (4.2) we know that $q_{N}, \tilde{q}_{N}: L^{p} \rightarrow L_{p}$ for any $p>2$. In addition we have

Lemma 9. Fix $N \geq 1$ and $p>2$. Then there exist positive constants $C_{p}$ and $L_{N, p}$ such that

$$
\begin{aligned}
\left\|q_{N}(\xi)\right\|_{L_{p}} \leq C_{p}(N+1)^{2} & \forall \xi \in L^{p}, \\
\left\|\tilde{q}_{N}(\xi)\right\|_{L_{p}} \leq C_{p}(N+1)^{2} & \forall \xi \in L^{p}
\end{aligned}
$$

and

$$
\left\|q_{N}(\xi)-q_{N}(\eta)\right\|_{L_{p}} \leq L_{N, p}\|\xi-\eta\|_{L^{p}} \quad \forall \xi, \eta \in L^{p} .
$$

Proof. The global bounds comes from (4.2):

$$
\begin{gathered}
\left\|q_{N}(\xi)\right\|_{L_{p}} \leq C_{p}\|\xi\|_{L^{p}}^{2} \Theta_{N}\left(\|\xi\|_{L^{p}}\right) \leq C_{p}(N+1)^{2}, \\
\left\|\tilde{q}_{N}(\xi)\right\|_{L_{p}} \leq C_{p}\|\xi\|_{L^{p}}^{2}\left|\Theta_{N}^{\prime}\left(\|\xi\|_{L^{p}}\right)\right| \leq C_{p}(N+1)^{2} .
\end{gathered}
$$

Let us now show that $q_{N}$ is a Lipschitz continuous function. The idea is to use the mean value theorem: we show that $q_{N}$ is Gâteaux differentiable in any point of $L^{p}$ and its derivative is bounded. The result will follow by

$$
\left\|q_{N}(\xi)-q_{N}(\eta)\right\|_{L_{p}} \leq \sup _{t \in[0,1]}\left\|D q_{N}(t \xi+(1-t) \eta)\right\|_{\mathcal{L}\left(L^{p} ; L_{p}\right)}\|\xi-\eta\|_{L^{p}}
$$

where $D q_{N}(\xi): h \rightarrow D_{h} q_{N}(\xi)$ is a linear and bounded operator from $L^{p}$ into $L_{p}$ defined as

$$
D_{h} q_{N}(\xi):=\lim _{\varepsilon \rightarrow 0} \frac{q_{N}(\xi+\varepsilon h)-q_{N}(\xi)}{\varepsilon}
$$

and

$$
\left\|D q_{N}(\xi)\right\|_{\mathcal{L}\left(L^{p} ; L_{p}\right)}=\sup _{\|h\|_{L^{p} \leq 1}}\left\|D_{h} q_{N}(\xi)\right\|_{L_{p}}
$$

By (4.12) we have

$$
D_{h} q_{N}(\xi)=q(\xi) D_{h} \Theta_{N}\left(\|\xi\|_{L^{p}}\right)+h(k * \xi) \Theta_{N}\left(\|\xi\|_{L^{p}}\right)+\xi(k * h) \Theta_{N}\left(\|\xi\|_{L^{p}}\right) .
$$

Since $D_{h}\left(\|\xi\|_{L^{p}}\right)=\|\xi\|_{L^{p}}^{1-p}\left\langle\xi|\xi|^{p-2}, h\right\rangle$ we get

$$
D_{h} \Theta_{N}\left(\|\xi\|_{L^{p}}\right)=\Theta_{N}^{\prime}\left(\|\xi\|_{L^{p}}\right)\|\xi\|_{L^{p}}^{1-p}\left\langle\xi|\xi|^{p-2}, h\right\rangle .
$$


Therefore, bearing in mind (2.17) and (4.9) we infer that

$$
\begin{aligned}
& \left\|D_{h} q_{N}(\xi)\right\|_{L_{p}} \\
& \leq\left|\Theta_{N}^{\prime}\left(\|\xi\|_{L^{p}}\right)\right|\|\xi\|_{L^{p}}^{1-p}\left|\left\langle\xi|\xi|^{p-2}, h\right\rangle\right|\|q(\xi)\|_{L_{p}} \\
& \quad+\left|\Theta_{N}\left(\|\xi\|_{L^{p}}\right)\right|\|h(k * \xi)\|_{L_{p}}+\left|\Theta_{N}\left(\|\xi\|_{L^{p}}\right)\right|\|\xi(k * h)\|_{L_{p}} \\
& \leq\left|\Theta_{N}^{\prime}\left(\|\xi\|_{L^{p}}\right)\right|\|h\|_{L^{p}}\|q(\xi)\|_{L_{p}} \\
& \quad+\left|\Theta_{N}\left(\|\xi\|_{L^{p}}\right)\right|\|h\|_{L^{p}}\|k * \xi\|_{L_{\infty}}+\left|\Theta_{N}\left(\|\xi\|_{L^{p}}\right)\right|\|\xi\|_{L^{p}}\|k * h\|_{L_{\infty}} \\
& \leq\|h\|_{L^{p}}\left\|\tilde{q}_{N}(\xi)\right\|_{L_{p}}+2 C_{p}\left|\Theta_{N}\left(\|\xi\|_{L^{p}}\right)\right|\|h\|_{L^{p}}\|\xi\|_{L^{p}} \\
& \leq C_{p}(N+1)^{2}\|h\|_{L^{p}}+2 C_{p}(N+1)\|h\|_{L^{p}} .
\end{aligned}
$$

Hence we get

$$
\sup _{\xi}\left\|D q_{N}(\xi)\right\|_{\mathcal{L}\left(L^{p} ; L_{p}\right)} \leq C_{p}(N+1)^{2}+2 C_{p}(N+1) .
$$

Thanks to (4.11) this proves (4.10).

We aim at proving the existence and uniqueness of the solution to the smoothed version of system (1.2) that is

$$
\left\{\begin{array}{l}
\frac{\partial \xi_{N}}{\partial t}(t, x)-\Delta \xi_{N}(t, x)+v_{N}(t, x) \cdot \nabla \xi_{N}(t, x) \Theta_{N}\left(\left\|\xi_{N}(t, \cdot)\right\|_{L^{p}}\right)=w(\mathrm{~d} x, \mathrm{~d} t) \\
\nabla \cdot v_{N}(t, x)=0 \\
\xi_{N}(t, x)=\nabla^{\perp} \cdot v_{N}(t, x) \\
\xi_{N}(0, x)=\xi_{0}(x)
\end{array}\right.
$$

Thanks to (4.1) and (4.6) this can be written in the Walsh formulation as

$$
\begin{aligned}
\xi_{N}(t, x)=\int_{D} g(t, x, y) \xi_{0}(y) \mathrm{d} y+\int_{0}^{t} \int_{D} \nabla_{y} g(t-s, x, y) & \cdot q_{N}\left(\xi_{N}(s, \cdot)\right)(y) \mathrm{d} y \mathrm{~d} s \\
& +\int_{0}^{t} \int_{D} g(t-s, x, y) w(\mathrm{~d} y, \mathrm{~d} s) .
\end{aligned}
$$

We have the following result.

Proposition 10. Let $N \geq 1, b>0$ in (2.23) and $p>2$. If $\xi_{0} \in L^{p}$, then there exists a unique solution $\xi_{N}$ to equation (4.13) which is an $\mathcal{F}_{t}$-adapted process whose paths belong to $C\left([0, T] ; L^{p}\right), \mathbb{P}$-a.s.

Proof. Since we are dealing with an additive noise, i.e. a noise which is independent of the unknown process $\xi$, we can work pathwise. In order to prove the existence and uniqueness result we appeal to the contraction principle. Let $\mathcal{B}$ denote the space of all $L^{p}$-valued $\mathcal{F}_{t}$-adapted stochastic processes $\eta(t, \cdot), t \in[0, T]$ such that the norm

$$
\|\eta\|_{\mathcal{B}}:=\sup _{t \in[0, T]}\|\eta(t, \cdot)\|_{L^{p}}
$$

is finite $\mathbb{P}$-a.s.

Define the operator $\mathcal{M}$ on $\mathcal{B}$ by

$$
\mathcal{M}\left(\xi_{N}\right)(t, x):=M_{0}(t, x)+\left(J q_{N}\left(\xi_{N}\right)\right)(t, x)+z(t, x),
$$

where

$$
M_{0}(t, x):=\int_{D} g(t, x, y) \xi_{0}(y) \mathrm{d} y
$$


and the other two terms are given respectively by (3.1) and (3.8). More precisely,

$$
\left(J q_{N}\left(\xi_{N}\right)\right)(t, x)=\int_{0}^{t} \int_{D} \nabla_{y} g(t-s, x, y) \cdot q_{N}\left(\xi_{N}(s, \cdot)\right)(y) \mathrm{d} y \mathrm{~d} s .
$$

Then

$$
\left\|\mathcal{M}\left(\xi_{N}\right)(t, \cdot)\right\|_{L^{p}}^{p} \leq C_{p}\left(\left\|M_{0}(t, \cdot)\right\|_{L^{p}}^{p}+\left\|\left(J q_{N}\left(\xi_{N}\right)\right)(t, \cdot)\right\|_{L^{p}}^{p}+\|z(t, \cdot)\|_{L^{p}}^{p}\right) .
$$

Using Young's inequality and (2.10), we infer that

$$
\left\|M_{0}\right\|_{\mathcal{B}}=\sup _{t \in[0, T]}\left\|M_{0}(t, \cdot)\right\|_{L^{p}} \leq \sup _{t \in[0, T]}\left(\|g(t, 0, \cdot)\|_{L^{1}}\left\|\xi_{0}\right\|_{L^{p}}\right)<\infty .
$$

By estimates (3.2) (with $\beta=1, \alpha=p$ ) and (4.8) we get

$$
\left\|\left(J q_{N}\left(\xi_{N}\right)\right)(t, \cdot)\right\|_{L^{p}} \leq \int_{0}^{t}(t-s)^{-\frac{1}{2}}\left\|q_{N}\left(\xi_{N}(s, \cdot)\right)\right\|_{L_{p}} \mathrm{~d} s \leq C_{p}(N+1)^{2} t^{\frac{1}{2}}
$$

and so $\left\|J q_{N}\left(\xi_{N}\right)\right\|_{\mathcal{B}}<\infty$. Finally, $\|z\|_{\mathcal{B}}<\infty \mathbb{P}$-a.s. by Lemma 7 . Thus $\mathcal{M}$ is an operator mapping the Banach space $\mathcal{B}$ into itself. It remains to prove that $\mathcal{M}$ is a contraction. From (3.2) with $\alpha=p$, $\beta=1$ and the Lipschitz result of Lemma 9 , we infer that

$$
\begin{aligned}
\| \mathcal{M}\left(\xi_{N}^{1}\right)(t, \cdot)-\mathcal{M} & \left(\xi_{N}^{2}\right)(t, \cdot)\left\|_{L^{p}} \leq C \int_{0}^{t}(t-s)^{-\frac{1}{2}}\right\| q_{N}\left(\xi_{N}^{1}(s, \cdot)\right)-q_{N}\left(\xi_{N}^{2}(s, \cdot)\right) \|_{L_{p}} d s \\
& \leq C L_{N, p} \int_{0}^{t}(t-s)^{-\frac{1}{2}}\left\|\xi_{N}^{1}(s, \cdot)-\xi_{N}^{2}(s, \cdot)\right\|_{L^{p}} \mathrm{~d} s \\
& \leq C L_{N, p}\left(\sup _{t \in[0, T]}\left\|\xi_{N}^{1}(t, \cdot)-\xi_{N}^{2}(t, \cdot)\right\|_{L^{p}}\right) \int_{0}^{t}(t-s)^{-\frac{1}{2}} \mathrm{~d} s \\
& \leq C_{N, p} T^{\frac{1}{2}}\left\|\xi_{N}^{1}-\xi_{N}^{2}\right\|_{\mathcal{B}}
\end{aligned}
$$

for every $t \in[0, T]$. If $T$ satisfies $C_{N, p} T^{\frac{1}{2}}<1$, then $\mathcal{M}$ is a contraction on $\mathcal{B}$. Hence the operator $\mathcal{M}$ admits a unique fixed point in the set $\left\{\xi \in \mathcal{B}: \xi(0, \cdot)=\xi_{0}\right\}$. Otherwise we choose $\tilde{t}>0$ such that $C_{N, p} \tilde{t}^{\frac{1}{2}}<1$ and we conclude the existence of a unique solution on the time interval $[0, \tilde{t}]$. Since $C_{N, p}$ does not depend on $\xi_{0}$, by a standard argument we construct a unique solution $\xi$ to the SPDE (4.13) by concatenation on every interval of lenght $\tilde{t}$ until we recover the time interval $[0, T]$.

In the following subsection we shall see that Proposition 10 provides uniqueness and local existence for the solution in Theorem 1, To gain the global existence we need a uniform estimate as proved in the following lemma, inspired by [14] and [15].

Let $z$ be the process defined in (3.8) and $\xi_{N}$ be the solution to equation (4.13). Let us set $\beta_{N}=$ $\xi_{N}-z$. Since the noise is independent on the unknown, $\beta_{N}$ satisfies the equation

$$
\frac{\partial}{\partial t} \beta_{N}=\Delta \beta_{N}-\nabla \cdot q_{N}\left(\beta_{N}+z\right)
$$

which can be written à la Walsh as

$$
\begin{aligned}
\beta_{N}(t, x)=\int_{D} g(t, x, y) \xi_{0}(y) \mathrm{d} y & \\
& +\int_{0}^{t} \int_{D} \nabla_{y} g(t-s, x, y) \cdot q_{N}\left(\beta_{N}(s, \cdot)+z(s, \cdot)\right)(y) \mathrm{d} y \mathrm{~d} s .
\end{aligned}
$$


The following result provides a uniform estimate for $\beta_{N}$. Notice that we shall work pathwise since the noise is additive.

Lemma 11. Let $b>0$ in (2.23) and $p>2$. If $\xi_{0} \in L^{p}$ then

$$
\sup _{N \geq 1} \sup _{t \in[0, T]}\left\|\beta_{N}(t, \cdot)\right\|_{L^{p}}^{p} \leq\left[\left\|\xi_{0}\right\|_{L^{p}}^{p}+C_{1}(z)\right] e^{C_{2}(z)}
$$

where $C_{1}(z)$ and $C_{2}(z)$ are given by

$$
C_{1}(z)=C_{p} T \sup _{t \in[0, T]}\|z(t, \cdot)\|_{L^{p}}^{2 p}
$$

and

$$
C_{2}(z)=C_{p} T\left(1+\sup _{t \in[0, T]}\|z(t, \cdot)\|_{L^{p}}^{2}\right)
$$

for some positive constant $C_{p}$.

Proof. As done before, we can show that a solution to (4.15) is a weak solution to the PDE (4.14) with initial condition $\beta_{N}(0, x)=\xi_{0}(x)$.

We consider the time evolution of the $L^{p}$-norm of $\beta_{N}(t, \cdot)$. Let us recall that when $b>0$ in (2.23), $z$ admits a modification with $\mathbb{P}$-a.s. space-time continuous trajectories; moreover from Proposition 10 we know that $\xi_{N} \in C\left([0, T] ; L^{p}\right) \mathbb{P}$-a.s. Hence, for sure, the solution $\beta_{N} \in C\left([0, T] ; L^{p}\right) \mathbb{P}$-a.s. for every $N \geq 1$. Actually, since the noise term has disappeared, $\beta_{N}$ is more regular than $\xi_{N}$ and $z$. Indeed, $\frac{\partial \beta_{N}}{\partial t}-\Delta \beta_{N}=-\nabla \cdot q_{N}\left(\xi_{N}\right)$ where $q_{N}\left(\xi_{N}\right)$ belongs at least to $L^{2}\left(0, T ; L_{2}\right)$ thanks to (4.8). Hence, according to a classical regularity result for parabolic equations (see e.g. [16, Chapter 4.4, Theorem 4.1]) we have that $\beta_{N} \in L^{2}\left(0, T ; W^{1}\right)$; hence $\nabla \beta_{N}$ exists. We use this fact in the following computations. Only at the end we will obtain an estimate involving $\nabla \beta_{N}$ which shows its regularity. This is a short way to prove our result. Otherwise one has to use Galerkin approximations and then pass to the limit.

From (4.14) we infer that

$$
\begin{aligned}
\frac{d}{d t}\left\|\beta_{N}(t, \cdot)\right\|_{L^{p}}^{p}= & p \int_{D}\left|\beta_{N}(t, x)\right|^{p-2} \beta_{N}(t, x) \frac{\partial}{\partial t} \beta_{N}(t, x) d x \\
= & p \int_{D}\left|\beta_{N}(t, x)\right|^{p-2} \beta_{N}(t, x) \Delta \beta_{N}(t, x) d x \\
& \quad-p \int_{D}\left|\beta_{N}(t, x)\right|^{p-2} \beta_{N}(t, x) \nabla \cdot q_{N}\left(\beta_{N}(t, \cdot)+z(t, \cdot)\right)(x) d x
\end{aligned}
$$

Integrating by parts the two latter integrals we obtain (writing for short $\beta_{N}(t)$ instead of $\beta_{N}(t, \cdot)$ )

$$
\begin{aligned}
\frac{d}{d t}\left\|\beta_{N}(t)\right\|_{L^{p}}^{p}+p(p-1)\left\|\left|\beta_{N}(t)\right|^{\frac{p-2}{2}} \nabla \beta_{N}(t)\right\|_{L^{2}}^{2} & \\
& =p(p-1)\left\langle\left|\beta_{N}(t)\right|^{p-2}, \nabla \beta_{N}(t) \cdot q_{N}\left(\beta_{N}(t)+z(t)\right)\right\rangle .
\end{aligned}
$$

We need to work on the latter term. Let us write the quadratic term $q_{N}\left(\beta_{N}(t)+z(t)\right)$ in the form $\Theta_{N}\left(\left\|\beta_{N}(t)+z(t)\right\|_{L^{p}}\right) k *\left(\beta_{N}(t)+z(t)\right)\left(\beta_{N}(t)+z(t)\right)=\Theta_{N}\left(\left\|\beta_{N}(t)+z(t)\right\|_{L^{p}}\right) k *\left(\beta_{N}(t)+z(t)\right) \beta_{N}(t)+$ $\Theta_{N}\left(\left\|\beta_{N}(t)+z(t)\right\|_{L^{p}}\right) k *\left(\beta_{N}(t)+z(t)\right) z(t)$; then using the basic property $\left\langle\left|\beta_{N}(t)\right|^{p-2} \beta_{N}(t), \nabla \beta_{N}(t)\right.$. 
$v(t)\rangle=0$ (where $v$ is a divergence free velocity field; this is obtained again by integration by parts, see for instance [2, Lemma 2.2]) we obtain

$$
\begin{aligned}
\frac{d}{d t}\left\|\beta_{N}(t)\right\|_{L^{p}}^{p} & +p(p-1)\left\|\left|\beta_{N}(t)\right|^{\frac{p-2}{2}} \nabla \beta_{N}(t)\right\|_{L^{2}}^{2} \\
& =p(p-1)\left\langle\Theta_{N}\left(\left\|\beta_{N}(t)+z(t)\right\|_{L^{p}}\right)\left|\beta_{N}(t)\right|^{p-2} z(t), \nabla \beta_{N}(t) \cdot\left[k *\left(\beta_{N}(t)+z(t)\right)\right]\right\rangle .
\end{aligned}
$$

Let us estimate the r.h.s., using Hölder's and Young's inequalities.

$$
\begin{aligned}
& \quad\left|\left\langle\Theta_{N}\left(\left\|\beta_{N}(t)+z(t)\right\|_{L^{p}}\right)\left|\beta_{N}(t)\right|^{p-2} z(t), \nabla \beta_{N}(t) \cdot\left[k *\left(\beta_{N}(t)+z(t)\right)\right]\right\rangle\right| \\
& \leq\left|\Theta_{N}\left(\left\|\beta_{N}(t)+z(t)\right\|_{L^{p}}\right)\right|\left\|\left|\beta_{N}(t)\right|^{\frac{p-2}{2}} \nabla \beta_{N}(t)\right\|_{L^{2}} \\
& \quad\left\|\left|\beta_{N}(t)\right|^{\frac{p-2}{2}} z(t)\right\|_{L^{2}}\left\|k *\left(\beta_{N}(t)+z(t)\right)\right\|_{L_{\infty}} \\
& \leq C_{p}\left\|\left|\beta_{N}(t)\right|^{\frac{p-2}{2}} \nabla \beta_{N}(t)\right\|_{L^{2}}\left\|\left|\beta_{N}(t)\right|^{\frac{p-2}{2}} z(t)\right\|_{L^{2}}\left\|\beta_{N}(t)+z(t)\right\|_{L^{p}} \quad \text { by (2.17) } \\
& \leq C_{p}\left\|\left|\beta_{N}(t)\right|^{\frac{p-2}{2}} \nabla \beta_{N}(t)\right\|_{L^{2}}\left\|\beta_{N}(t)\right\|_{L^{p}}^{\frac{p-2}{2}}\|z(t)\|_{L^{p}}\left(\left\|\beta_{N}(t)\right\|_{L^{p}}+\|z(t)\|_{L^{p}}\right) \\
& \leq \frac{1}{2}\left\|\left|\beta_{N}(t)\right|^{\frac{p-2}{2}} \nabla \beta_{N}(t)\right\|_{L^{2}}^{2}+C_{p}\left\|\beta_{N}(t)\right\|_{L^{p}}^{p}\|z(t)\|_{L^{p}}^{2} \\
& \quad+C_{p}\left\|\beta_{N}(t)\right\|_{L^{p}}^{p}+C_{p}\|z(t)\|_{L^{p}}^{2 p} .
\end{aligned}
$$

Coming back to equation (4.16), we have obtained that

$$
\begin{aligned}
\frac{d}{d t}\left\|\beta_{N}(t)\right\|_{L^{p}}^{p}+\frac{p(p-1)}{2}\left\|\left|\beta_{N}(t)\right|^{\frac{p-2}{2}} \nabla \beta_{N}(t)\right\|_{L^{2}}^{2} & \\
& \leq C_{p}\left(1+\|z(t)\|_{L^{p}}^{2}\right)\left\|\beta_{N}(t)\right\|_{L^{p}}^{p}+C_{p}\|z(t)\|_{L^{p}}^{2 p} .
\end{aligned}
$$

Using Gronwall lemma on the inequality

we obtain

$$
\frac{d}{d t}\left\|\beta_{N}(t)\right\|_{L^{p}}^{p} \leq C_{p}\left(1+\|z(t)\|_{L^{p}}^{2}\right)\left\|\beta_{N}(t)\right\|_{L^{p}}^{p}+C_{p}\|z(t)\|_{L^{p}}^{2 p}
$$

$$
\begin{aligned}
\left\|\beta_{N}(t)\right\|_{L^{p}}^{p} & \leq\left\|\xi_{0}\right\|_{L^{p}}^{p} e^{C_{p} \int_{0}^{t}\left(1+\|z(s)\|_{L^{p}}^{2}\right) \mathrm{d} s}+C_{p} \int_{0}^{t} e^{C_{p} \int_{r}^{t}\left(1+\|z(s)\|_{L^{p}}^{2}\right) \mathrm{d} s}\|z(r)\|_{L^{p}}^{2 p} \mathrm{~d} r \\
& \leq e^{C_{p} T\left(1+\sup _{0 \leq s \leq T}\|z(s)\|_{L^{p}}^{2}\right)}\left(\left\|\xi_{0}\right\|_{L^{p}}^{p}+C_{p} T \sup _{0 \leq r \leq T}\|z(r)\|_{L^{p}}^{2 p}\right) .
\end{aligned}
$$

Integrating in time (4.17), we obtain that $\left|\beta_{N}\right|^{\frac{p-2}{2}} \nabla \beta_{N} \in L^{2}\left(0, T ; L^{2}\right)$ which is the regularity we expected.

4.2. Existence and uniqueness of the solution to (4.4). We go back to the original equation (1.2) in the form given by (4.4) and prove the existence and uniqueness result stated in Theorem 1 .

Proof of Theorem 1. Pathwise uniqueness is provided in a classical way by a stopping time argument. More precisely, suppose that $\xi^{1}$ and $\xi^{2}$ are two solutions to equation (1.2). Both satisfy (4.4) thanks to the equivalence between the formulations (4.3) and (4.4). Let $p>2$; let us define the stopping times

$$
\tau_{N}^{i}:=\inf \left\{t \geq 0:\left\|\xi^{i}(t, \cdot)\right\|_{L^{p}} \geq N\right\} \wedge T, \quad i=1,2,
$$


for every $N \geq 1$ and let us set $\tau_{N}^{*}:=\tau_{N}^{1} \wedge \tau_{N}^{2}$. Setting $\xi_{N}^{i}(t)=\xi^{i}\left(t \wedge \tau_{N}^{*}\right)$ for $i=1,2$, for all $t \in[0, T]$ we have that the processes $\xi_{N}^{1}$ and $\xi_{N}^{2}$ satisfy (4.13); hence, by the uniqueness result given by Proposition 10. $\xi_{N}^{1}=\xi_{N}^{2} \mathbb{P}$-a.s. for all $t \in[0, T]$, that is $\xi^{1}=\xi^{2}$ on $\left[0, \tau_{N}^{*}\right) \mathbb{P}$-a.s. Since $\tau_{N}^{*}$ converges $\mathbb{P}$-a.s. to $T$, as $N$ tends to infinity, we deduce $\xi^{1}=\xi^{2} \mathbb{P}$-a.s for every $t \in[0, T]$.

Let us now prove the existence of the solution in $[0, T]$. Let $p>2$; let us define the stopping time

$$
\sigma_{N}:=\inf \left\{t \geq 0:\left\|\xi_{N}(t, \cdot)\right\|_{L^{p}} \geq N\right\} \wedge T
$$

for every $N \geq 1$. In Proposition 10 we have shown the global existence and uniqueness of the solution $\xi_{N}$ to the truncated problem (4.13). By uniqueness it follows that, given $M>N$ we have $\xi_{N}(t, \cdot)=\xi_{M}(t, \cdot)$ for $t \leq \sigma_{N}$; so we can define a process $\xi$ by $\xi(t, \cdot)=\xi_{N}(t, \cdot)$ for $t \in\left[0, \sigma_{N}\right]$. Set $\sigma_{\infty}:=\sup _{N \geq 1} \sigma_{N}$, then Proposition 10 tells us that we have constructed a solution to (4.13) in the random interval $\left[0, \sigma_{\infty}\right)$, and it is unique. To conclude, we just need to prove that

$$
\sigma_{\infty}=T \quad \mathbb{P}-\text { a.s. }
$$

that is equivalent to verify that

$$
\lim _{N \rightarrow \infty} \mathbb{P}\left(\sigma_{N}<T\right)=0
$$

By Lemma 11 we have that, for every $N \geq 1$,

$$
\sup _{t \in[0, T]} \log \left\|\beta_{N}(t, \cdot)\right\|_{L^{p}} \leq \frac{1}{p} \log \left(\left\|\xi_{0}\right\|_{L^{p}}^{p}+C_{1}(z)\right)+\frac{C_{2}(z)}{p}
$$

and $\mathbb{E}\left[C_{1}(z)\right], \mathbb{E}\left[C_{2}(z)\right]$ are finite, according to Lemma 7 . Hence, for all $N \geq 1$,

$$
\mathbb{E}\left[\sup _{t \in[0, T]} \log \left\|\beta_{N}(t, \cdot)\right\|_{L^{p}}\right] \leq C_{p, T}\left(1+\log \left\|\xi_{0}\right\|_{L^{p}}^{p}\right)<\infty
$$

by means of Jensen's inequality. By Chebychev's inequality, it follows that

$$
\begin{aligned}
\mathbb{P}\left(\sigma_{N}<T\right) & =\mathbb{P}\left(\sup _{t \in[0, T]}\left\|\xi_{N}(t, \cdot)\right\|_{L^{p}}>N\right) \\
& \leq \mathbb{P}\left(\sup _{t \in[0, T]}\left\|\beta_{N}(t, \cdot)\right\|_{L^{p}}>\frac{N}{2}\right)+\mathbb{P}\left(\sup _{t \in[0, T]}\|z(t, \cdot)\|_{L^{p}}>\frac{N}{2}\right) \\
& \leq \frac{1}{\log \left(\frac{N}{2}\right)} \mathbb{E}\left[\sup _{t \in[0, T]} \log \left\|\beta_{N}(t, \cdot)\right\|_{L^{p}}\right]+\frac{2}{N} \mathbb{E}\left[\sup _{t \in[0, T]}\|z(t, \cdot)\|_{L^{p}}\right] \\
& \leq \frac{C_{p, T}\left(1+\log \left\|\xi_{0}\right\|_{L^{p}}^{p}\right)}{\log N}+\frac{\hat{C}_{p, T}}{N},
\end{aligned}
$$

for some constant $C_{p, T}$ and $\hat{C}_{p, T}$. Then we obtain that $\lim _{N \rightarrow \infty} \mathbb{P}\left(\sigma_{N}<T\right)=0$.

Now, we assume $\xi_{0}$ to be continuous; then the solution $\xi$ given by (4.4) is the sum of three terms. The first one, $\int_{D} g(t, x, y) \xi_{0}(y) \mathrm{d} y$ is continuous by the properties of $g$ (see Theorem $4(i i)$ ). As regards the second one, since $\xi_{0} \in C(D)$, then $\xi_{0} \in L^{\tilde{p}}$ for any $\tilde{p}$. Choosing a value of $\tilde{p}>4$, we find that $q(\xi) \in C\left([0, T] ; L_{\tilde{p}}\right)$ and Lemma $6(i i)$ provides that $J q(\xi) \in C([0, T] \times D)$. Finally the third term is continuous thanks to Lemma 7 . 


\section{Malliavin Calculus for the 2D Navier-Stokes equations in the vorticity FORMULATION}

We can use the framework of the Malliavin calculus in the setting introduced in Section 2.4, namely the underlying Gaussian space on which to perform Malliavin calculus is given by the isonormal Gaussian process on the Hilbert space $\mathcal{H}_{T}$. We recall here some basic facts about the Malliavin calculus. For full details we refer to [24].

A $\mathcal{F}$-measurable real valued random variable $F$ is said to be cylindrical if it can be written as

$$
F=f\left(W\left(\phi^{1}\right), \ldots, W\left(\phi^{n}\right)\right),
$$

where $\phi^{i} \in \mathcal{H}_{T}$ and $f: \mathbb{R}^{n} \rightarrow \mathbb{R}$ is a $C^{\infty}$ bounded function. The set of cylindrical random variables is denoted by $\mathcal{S}$. The Malliavin derivative of $F \in \mathcal{S}$ is the stochastic process $D F=\left\{D_{\sigma} F, \sigma \in \mathcal{H}_{T}\right\}$ given by

$$
D F=\sum_{i=1}^{n} \phi^{i} \frac{\partial f}{\partial x_{i}}\left(W\left(\phi^{1}\right), \ldots, W\left(\phi^{n}\right)\right) .
$$

The operator $D$ is closable from $\mathcal{S}$ into $L^{p}\left(\Omega, \mathcal{H}_{T}\right)$. We denote by $\mathbb{D}^{1, p}\left(\mathcal{H}_{T}\right)$ the closure of the class of cylindrical random variables with respect to the norm

$$
\|F\|_{1, p}=\left(\mathbb{E}\left(|F|^{p}\right)+\mathbb{E}\|D F\|_{\mathcal{H}_{T}}^{p}\right)^{\frac{1}{p}} .
$$

We also introduce the localized spaces; a random variable $F$ belongs to $\mathbb{D}_{\text {loc }}^{1, p}\left(\mathcal{H}_{T}\right)$ if there exists a sequence of sets $\Omega_{n} \subset \Omega$ and a sequence of random variables $F_{n} \in \mathbb{D}^{1, p}\left(\mathcal{H}_{T}\right)$ such that $\Omega_{n} \uparrow \Omega$ almost surely and $F=F_{n}$ on $\Omega_{n}$. Then for any $n$ we set $D F=D F_{n}$ on $\Omega_{n}$. We refer to $\left(\Omega_{n}, F_{n}\right)$ as a localizing sequence for $F$.

The following key result stems from [24, Theorem 2.1.3]:

Theorem 12. Let $F$ be a $\mathcal{F}$-measurable random variable such that $F \in \mathbb{D}_{\text {loc }}^{1,1}\left(\mathcal{H}_{T}\right)$ and

$$
\|D F\|_{\mathcal{H}_{T}}>0, \quad \mathbb{P}-\text { a.s. }
$$

Then the law of $F$ has a density with respect to the Lebesgue measure on $\mathbb{R}$.

In order to prove the assumption $F \in \mathbb{D}_{\text {loc }}^{1,1}\left(\mathcal{H}_{T}\right)$ in our setting, we shall work on a sequence of smoothed processes and use the following result (see [24, Lemma 1.5.3]).

Proposition 13. Let $\left\{F_{k}\right\}_{k}$ be a sequence of random variables in $\mathbb{D}^{1, p}$ for some $p>1$. Assume that the sequence $F_{k}$ converges to $F$ in $L^{p}(\Omega)$ and that

$$
\sup _{k}\left\|F_{k}\right\|_{1, p}<\infty .
$$

Then $F$ belongs to $\mathbb{D}^{1, p}$.

We use these results for the random variable $\xi(t, x)$, solution to equation (4.4) and the random variable $\xi_{N}(t, x)$ solution to equation (4.13). More precisely, by means of Proposition 13, in Section 5.1. we show that $\xi_{N}(t, x) \in \mathbb{D}^{1, p}$; hence $\xi(t, x) \in \mathbb{D}_{\text {loc }}^{1, p}$. In Subsection 5.2 we prove that $\xi_{N}(t, x)$ satisfies assumption (5.1) of Theorem 12. The same condition holds for $\xi(t, x)$ as we shall see in Subsection 5.3 . 
5.1. Malliavin analysis of the truncated equation. In order to show that $\xi_{N}(t, x) \in \mathbb{D}^{1, p}$ we use Proposition 13. We introduce a Picard approximation sequence $\left\{\xi_{N}^{k}\right\}_{k}$ for $\xi_{N}$ and we show that as $k \rightarrow$ $+\infty$, the sequence $\xi_{N}^{k}(t, x)$ converges to $\xi_{N}(t, x)$ in $L^{p}(\Omega)$ (for $N \geq 1$ fixed) and $\sup _{k}\left\|\xi_{N}^{k}(t, x)\right\|_{1, p}<\infty$ uniformly in $(t, x) \in[0, T] \times D$. A similar argument has been used in [5] for the Cahn-Hilliard stochastic equation and in [21] for the one dimensional Burgers equation. Let us point out that the smoothness of the density cannot be obtained via this location argument, since this procedure does not provide the boundedness of the Malliavin derivatives of every order.

First, we need to improve the result of Proposition [10. This is done in the following theorem, whose proof provides the approximating sequence $\left\{\xi_{N}^{k}\right\}_{k}$ of the Picard scheme.

Theorem 14. Fix $N \geq 1$ and $p>4$. If $b>0$ in (2.23) and $\xi_{0}$ is a continuous function on $D$, then the solution process $\xi_{N}$ to (4.13) satisfies

$$
\sup _{(t, x) \in[0, T] \times D} \mathbb{E}\left|\xi_{N}(t, x)\right|^{p}<\infty .
$$

Proof. Let us consider a Picard iteration scheme for equation (4.13). We define

$$
\xi_{N}^{0}(t, x)=\int_{D} \xi_{0}(y) g(t, x, y) \mathrm{d} y
$$

and recursively, for $k \geq 0$

$$
\xi_{N}^{k+1}(t, x)=\xi_{N}^{0}(t, x)+z(t, x)+\left(J q_{N}\left(\xi_{N}^{k}\right)\right)(t, x)
$$

with $z$ and $J q_{N}\left(\xi_{N}^{k}\right)$ defined respectively as in (3.8) and (3.1). Notice that every term in (5.5) is well defined. The well posedness of the stochastic term follows from (2.24). On the other hand (3.4), (4.8) and Proposition 10 provide the well posedness of the non linear term.

For every $(t, x) \in[0, T] \times D$, from Lemma $6($ (ii) (for $\gamma=p$, provided $p>4$ ) and Lemma 9 we get

$$
\mathbb{E}\left|\xi_{N}^{k+1}(t, x)-\xi_{N}^{k}(t, x)\right|^{p} \leq C_{N, T, p} \int_{0}^{t} \int_{D} \mathbb{E}\left|\xi_{N}^{k}(s, y)-\xi_{N}^{k-1}(s, y)\right|^{p} \mathrm{~d} y \mathrm{~d} s .
$$

For every $k \geq 1$ we set

$$
\varphi_{k}(t, x)=\mathbb{E}\left|\xi_{N}^{k}(t, x)-\xi_{N}^{k-1}(t, x)\right|^{p} .
$$

Then $\varphi_{1} \in L^{1}([0, T] \times D)$ for the same considerations made for the well posedness of (5.5). From (5.6), by iteration (see e.g. [24, Theorem 2.4.3]) and [21, Proposition 5.1]) we get

$$
\begin{aligned}
\varphi_{k+1}(t, x) & \leq C_{N, p, T} \int_{0}^{t} \int_{D} \varphi_{k}(s, y) \mathrm{d} y \mathrm{~d} s \leq \ldots \\
& \leq C_{N, p, T}^{k} \int_{0}^{t} \int_{D}\left[\int_{0}^{s_{1}} \int_{D} \cdots\left(\int_{0}^{s_{k-1}} \int_{D} \varphi_{1}\left(s_{k}, y_{k}\right) \mathrm{d} y_{k} \mathrm{~d} s_{k}\right) \cdots\right] \mathrm{d} y_{1} \mathrm{~d} s_{1} \\
& =C_{N, p, T}^{k}|D|^{k-1} \frac{t^{k-1}}{(k-1) !} \int_{0}^{t} \int_{D} \varphi_{1}\left(s_{k}, y_{k}\right) \mathrm{d} y_{k} \mathrm{~d} s_{k} .
\end{aligned}
$$

This allows us to infer that

$$
\sum_{k=1}^{\infty} \sup _{(t, x) \in[0, T] \times D} \varphi_{k+1}(t, x) \leq\left(\sum_{k=1}^{\infty} C_{N, p, T}^{k}|D|^{k-1} \frac{t^{k-1}}{(k-1) !}\right) \int_{0}^{t} \int_{D} \varphi_{1}(s, y) \mathrm{d} y \mathrm{~d} s .
$$


Since the latter series converges, we deduce that

$$
\sum_{k=1}^{\infty} \sup _{(t, x) \in[0, T] \times D} \mathbb{E}\left|\xi_{N}^{k+1}(t, x)-\xi_{N}^{k}(t, x)\right|^{p}<\infty .
$$

This implies that, as $k$ tends to infinity, the sequence $\xi_{N}^{k}(t, x)$ converges in $L^{p}(\Omega)$, uniformly in time and space, to a stochastic process $\xi_{N}(t, x)$. Moreover,

$$
\sup _{k} \sup _{(t, x) \in[0, T] \times D} \mathbb{E}\left|\xi_{N}^{k}(t, x)\right|^{p}<\infty .
$$

It follows that the process $\xi_{N}(t, x)$ is adapted and satisfies (4.13) and (5.3).

Let us study the Malliavin derivative of the solution $\xi_{N}$ to the smoothed equation (4.13). Let us recall that the underlying Gaussian space on which to perform Malliavin calculus is given by the isonormal Gaussian process on the Hilbert space $\mathcal{H}_{T}:=L^{2}\left(0, T ; L_{Q}^{2}\right)$ which can be associated to the noise coloured in space by the covariance $Q$.

In this part, to keep things as simple as possible, in some points we go back to the notation involving $\xi_{N}$ and $v_{N}$ instead of $q_{N}\left(\xi_{N}\right)$, with $v_{N}=k * \xi_{N}$. Keeping in mind the definition of $\tilde{q}_{N}(\xi)$ given (4.7) we state the following result.

Theorem 15. Fix $N \geq 1$. Suppose that $b>0$ in (2.23) and $\xi_{0}$ is a continuous function on $D$. Then for all $(t, x) \in[0, T] \times D$ the solution $\xi_{N}(t, x)$ to (4.13) belongs to $\mathbb{D}^{1, p}$ for every $p>4$ and its Malliavin derivative satisfies the equation

$$
\begin{aligned}
& D_{r, z} \xi_{N}(t, x)=g(t-r, x, z) \mathbf{1}_{[0, t]}(r) \\
& +\int_{r}^{t} \int_{D} \nabla_{y} g(t-s, x, y) \cdot v_{N}(s, y) \Theta_{N}\left(\left\|\xi_{N}(s, \cdot)\right\|_{L^{p}}\right) D_{r, z} \xi_{N}(s, y) \mathrm{d} y \mathrm{~d} s \\
& +\int_{r}^{t} \int_{D}\left(\nabla_{y} g(t-s, x, y) \cdot \int_{D} k(y-\alpha) D_{r, z} \xi_{N}(s, \alpha) \mathrm{d} \alpha\right) \\
& \quad \Theta_{N}\left(\left\|\xi_{N}(s, \cdot)\right\|_{L^{p}}\right) \xi_{N}(s, y) \mathrm{d} y \mathrm{~d} s \\
& +\int_{r}^{t} \int_{D} \nabla_{y} g(t-s, x, y) \cdot \tilde{q}_{N}\left(\xi_{N}(s, \cdot)\right)(y) \\
& \quad p\left\|\xi_{N}(s, \cdot)\right\|_{L^{p}}^{1-p}\left(\int_{D}\left|\xi_{N}(s, \beta)\right|^{p-2} \xi_{N}(s, \beta) D_{r, z} \xi_{N}(s, \beta) \mathrm{d} \beta\right) \mathrm{d} y \mathrm{~d} s
\end{aligned}
$$

if $r \leq t$, and $D_{r, z} \xi_{N}(t, x)=0$ if $r>t$.

Proof. The proof of this part is based on Proposition 13, Let us consider the Picard approximation sequence $\left\{\xi_{N}^{k}(t, x)\right\}_{k}$ defined in (5.4) -(5.5); ; given the convergence (as $\left.k \rightarrow+\infty\right)$ obtained in the proof of Theorem [14, it is sufficient to show that

$$
\sup _{k} \sup _{(t, x) \in[0, T] \times D} \mathbb{E}\left\|D \xi_{N}^{k}(t, x)\right\|_{\mathcal{H}_{T}}^{p}<+\infty
$$

in order to prove that $\xi_{N}(t, x) \in \mathbb{D}^{1, p}$. Since $\xi_{N}^{0}$ is deterministic, it belongs to $\mathbb{D}^{1, p}$ and its Malliavin derivative is zero. Let us suppose that, for $k \geq 1$ and $p>4, \xi_{N}^{k}(t, x) \in \mathbb{D}^{1, p}$ for every $(t, x) \in[0, T] \times D$ and

$$
\sup _{(t, x) \in[0, T] \times D} \mathbb{E}\left\|D \xi_{N}^{k}(t, x)\right\|_{\mathcal{H}_{T}}^{p}<\infty .
$$


Applying the operator $D$ to equation (5.5) we obtain that the Malliavin derivative of $\xi_{N}^{k}(t, x)$ satisfies the equation (for more details see for instance [7, Proposition 2.15 and Proposition 2.16] and [24, Proposition 1.3.2])

$$
\begin{aligned}
& D_{r, z} \xi_{N}^{k+1}(t, x)=g(t-r, x, z) \mathbf{1}_{[0, t]}(r) \\
& +\int_{r}^{t} \int_{D} \nabla_{y} g(t-s, x, y) \cdot v_{N}^{k}(s, y) \Theta_{N}\left(\left\|\xi_{N}^{k}(s, \cdot)\right\|_{L^{p}}\right) D_{r, z} \xi_{N}^{k}(s, y) \mathrm{d} y \mathrm{~d} s \\
& +\int_{r}^{t} \int_{D}\left(\nabla_{y} g(t-s, x, y) \cdot \int_{D} k(y-\alpha) D_{r, z} \xi_{N}^{k}(s, \alpha) \mathrm{d} \alpha\right) \\
& \quad \Theta_{N}\left(\left\|\xi_{N}^{k}(s, \cdot)\right\|_{L^{p}}\right) \xi_{N}^{k}(s, y) \mathrm{d} y \mathrm{~d} s \\
& +\int_{r}^{t} \int_{D} \nabla_{y} g(t-s, x, y) \cdot \tilde{q}_{N}\left(\xi_{N}^{k}(s, \cdot)\right)(y) \\
& \quad p\left\|\xi_{N}^{k}(s, \cdot)\right\|_{L^{p}}^{1-p}\left(\int_{D}\left|\xi_{N}^{k}(s, \beta)\right|^{p-2} \xi_{N}^{k}(s, \beta) D_{r, z} \xi_{N}^{k}(s, \beta) \mathrm{d} \beta\right) \mathrm{d} y \mathrm{~d} s .
\end{aligned}
$$

We analyze the three integrals in the r.h.s. Let us set for simplicity

$$
\begin{gathered}
I_{1}(r, z):=\int_{r}^{t} \int_{D} \nabla_{y} g(t-s, x, y) \cdot v_{N}^{k}(s, y) \Theta_{N}\left(\left\|\xi_{N}^{k}(s, \cdot)\right\|_{L^{p}}\right) D_{r, z} \xi_{N}^{k}(s, y) \mathrm{d} y \mathrm{~d} s \\
I_{2}(r, z):=\int_{r}^{t} \int_{D}\left(\nabla_{y} g(t-s, x, y) \cdot \int_{D} k(y-\alpha) D_{r, z} \xi_{N}^{k}(s, \alpha) \mathrm{d} \alpha\right) \\
\Theta_{N}\left(\left\|\xi_{N}^{k}(s, \cdot)\right\|_{L^{p}}\right) \xi_{N}^{k}(s, y) \mathrm{d} y \mathrm{~d} s \\
I_{3}(r, z):=\int_{r}^{t} \int_{D} \nabla_{y} g(t-s, x, y) \cdot \tilde{q}_{N}\left(\xi_{N}^{k}(s, \cdot)\right)(y) \\
\left(p\left\|\xi_{N}^{k}(s, \cdot)\right\|_{L^{p}}^{1-p} \int_{D}\left|\xi_{N}^{k}(s, \beta)\right|^{p-2} \xi_{N}^{k}(s, \beta) D_{r, z} \xi_{N}^{k}(s, \beta) \mathrm{d} \beta\right) \mathrm{d} y \mathrm{~d} s .
\end{gathered}
$$

Then

$$
\mathbb{E}\left\|D \xi_{N}^{k+1}(t, x)\right\|_{\mathcal{H}_{T}}^{p} \leq C_{p}\left(\left\|g(t-\cdot, x, \cdot) \mathbf{1}_{[0, t]}(\cdot)\right\|_{\mathcal{H}_{T}}^{p}+\sum_{i=1}^{3} \mathbb{E}\left\|I_{i}\right\|_{\mathcal{H}_{T}}^{p}\right)
$$

Let us estimate the various terms in (5.14). By the definition of $\mathcal{H}_{T}$ and (2.24), we get

$$
\left\|g(t-\cdot, x, \cdot) \mathbf{1}_{[0, t]}(\cdot)\right\|_{\mathcal{H}_{T}}^{p}=\left(\|g(t-\cdot, x, \cdot)\|_{\mathcal{H}_{t}}^{2}\right)^{\frac{p}{2}} \leq\left(\frac{1}{2(2 \pi)^{2}} \sum_{k \in \mathbb{Z}_{0}^{2}}|k|^{-2-2 b}\right)^{\frac{p}{2}}<\infty
$$


Minkowski's and Hölder's inequalities imply that

$$
\begin{aligned}
& \mathbb{E}\left\|I_{1}\right\|_{\mathcal{H}_{T}}^{p} \leq \mathbb{E}\left[\int_{0}^{t} \int_{D}\left|\nabla_{y} g(t-s, x, y) \cdot v_{N}^{k}(s, y) \Theta_{N}\left(\left\|\xi_{N}^{k}(s, \cdot)\right\|_{L^{p}}\right)\right|\left\|D \xi_{N}^{k}(s, y)\right\|_{\mathcal{H}_{T}} \mathrm{~d} y \mathrm{~d} s\right]^{p} \\
& \leq \mathbb{E}\left[\int_{0}^{t}\left|\Theta_{N}\left(\left\|\xi_{N}^{k}(s, \cdot)\right\|_{L^{p}}\right)\right|\left\|\nabla_{y} g(t-s, x, \cdot)\right\|_{L^{\frac{p}{p-1}}}\right. \\
& \left.\qquad\left(\int_{D}\left|v_{N}^{k}(s, y)\right|^{p}\left\|D \xi_{N}^{k}(s, y)\right\|_{\mathcal{H}_{T}}^{p} \mathrm{~d} y\right)^{\frac{1}{p}} \mathrm{~d} s\right]^{p} \\
& \leq \mathbb{E}\left[\int_{0}^{t}\left|\Theta_{N}\left(\left\|\xi_{N}^{k}(s, \cdot)\right\|_{L^{p}}\right)\right|\left\|\nabla_{y} g(t-s, x, \cdot)\right\|_{L^{\frac{p}{p-1}}}\right. \\
& \left.\leq C_{N}\left(\int_{0}^{t} \int_{D}\left|\nabla_{y} g(t-s, x, y)\right|^{\frac{p}{p-1}} \mathrm{~d} y \mathrm{~d} s\right)^{p-1}(s, \cdot)\left\|_{L_{\infty}}\right\| D \xi_{N}^{k}(s, \cdot) \|_{L^{p}\left(D ; \mathcal{H}_{T}\right)} \mathrm{d} s\right]^{p} \\
& \mathbb{E}\left[\int_{0}^{t}\left\|D \xi_{N}^{k}(s, \cdot)\right\|_{L^{p}(D ; \mathcal{H} T)}^{p} \mathrm{~d} s\right] \quad \text { by (2.17) } \\
& \leq C_{N} t^{\frac{p}{2}-2} \int_{0}^{t} \int_{D} \mathbb{E}\left\|D \xi_{N}^{k}(s, y)\right\|_{\mathcal{H}_{T}}^{p} \mathrm{~d} y \mathrm{~d} s \quad \text { by (2.9) provided } p>4 .
\end{aligned}
$$

As regards the term $I_{2}$ using Fubini's Theorem, Minkowski's and Hölder's inequalities we have

$$
\begin{aligned}
& \mathbb{E}\left\|I_{2}\right\|_{\mathcal{H}_{T}}^{p}=\mathbb{E} \| \int_{r}^{t} \int_{D}\left(\int_{D} \nabla_{y} g(t-s, x, y) \cdot k(y-\alpha) \xi_{N}^{k}(s, y) \mathrm{d} y\right) \Theta_{N}\left(\left\|\xi_{N}^{k}(s, \cdot)\right\|_{L^{p}}\right) D \xi_{N}^{k}(s, \alpha) \mathrm{d} \alpha \mathrm{d} s \|_{\mathcal{H}_{T}}^{p} \\
& \leq \mathbb{E}\left[\int_{0}^{t} \int_{D} \mid\left(\int_{D} \nabla_{y} g(t-s, x, y) \cdot k(y-\alpha) \xi_{N}^{k}(s, y) \mathrm{d} y\right)\right. \\
& \leq\left.\Theta_{N}\left(\left\|\xi_{N}^{k}(s, \cdot)\right\|_{L^{p}}\right) \mid\left\|D \xi_{N}^{k}(s, \alpha)\right\|_{\mathcal{H}_{T}} \mathrm{~d} \alpha \mathrm{d} s\right]^{p} \\
& \leq\left.\mathbb{E} \int_{0}^{t} \int_{D}\left\|\nabla_{y} g(t-s, x, \cdot) \cdot k(\cdot-\alpha)\right\|_{L^{\frac{p}{p-1}}\left\|\xi_{N}^{k}(s, \cdot)\right\|_{L^{p}}}\left|\Theta_{N}\left(\left\|\xi_{N}^{k}(s, \cdot)\right\|_{L^{p}}\right)\right|\left\|D \xi_{N}^{k}(s, \alpha)\right\|_{\mathcal{H}_{T}} \mathrm{~d} \alpha \mathrm{d} s\right]^{p} \\
& \leq C_{N} \mathbb{E}\left[\int_{0}^{t} \int_{D}\left\|\nabla_{y} g(t-s, x, \cdot) \cdot k(\cdot-\alpha)\right\|_{L^{\frac{p}{p-1}}}\left\|D \xi_{N}^{k}(s, \alpha)\right\|_{\mathcal{H}_{T}} \mathrm{~d} \alpha \mathrm{d} s\right]^{p} \\
& \leq C_{N} \mathbb{E}\left[\int_{0}^{t}\left(\int_{D} \int_{D}\left|\nabla_{y} g(t-s, x, y) \cdot k(y-\alpha)\right|^{\frac{p}{p-1}} \mathrm{~d} y \mathrm{~d} \alpha\right)^{\frac{p-1}{p}}\right. \\
&\left.\left\|D \xi_{N}^{k}(s, \cdot)\right\|_{L^{p}\left(D ; \mathcal{H}_{T}\right)} \mathrm{d} s\right]^{p} .
\end{aligned}
$$


By means of Fubini's Theorem, if $p>4$, we can estimate the inner integral

$$
\begin{aligned}
\int_{D} \int_{D} \mid & \left.\nabla_{y} g(t-s, x, y) \cdot k(y-\alpha)\right|^{\frac{p}{p-1}} \mathrm{~d} y \mathrm{~d} \alpha \\
& \leq \int_{D} \int_{D}\left|\nabla_{y} g(t-s, x, y)\right|^{\frac{p}{p-1}}|k(y-\alpha)|^{\frac{p}{p-1}} \mathrm{~d} y \mathrm{~d} \alpha \\
& =\int_{D}\left|\nabla_{y} g(t-s, x, y)\right|^{\frac{p}{p-1}}\left(\int_{D}|k(y-\alpha)|^{\frac{p}{p-1}} \mathrm{~d} \alpha\right) \mathrm{d} y \\
& \leq C \int_{D}\left|\nabla_{y} g(t-s, x, y)\right|^{\frac{p}{p-1}} \mathrm{~d} y \quad \text { by Lemma } 5 \text { and Remark 1 } \\
& \leq C_{p}(t-s)^{-\frac{3}{2}\left(\frac{p}{p-1}\right)+1 \quad \text { by (2.8) }},
\end{aligned}
$$

obtaining

$$
\begin{aligned}
\mathbb{E}\left\|I_{2}\right\|_{\mathcal{H}_{T}}^{p} & \leq C_{N, p} \mathbb{E}\left[\int_{0}^{t}(t-s)^{-\frac{p+2}{2 p}}\left\|D \xi_{N}^{k}(s, \cdot)\right\|_{L^{p}\left(D ; \mathcal{H}_{T}\right)} \mathrm{d} s\right]^{p} \\
& \leq C_{N, p}\left(\int_{0}^{t}(t-s)^{\frac{p+2}{2(1-p)}} \mathrm{d} s\right)^{p-1} \mathbb{E}\left[\int_{0}^{t}\left\|D \xi_{N}^{k}(s, \cdot)\right\|_{L^{p}\left(D ; \mathcal{H}_{T}\right)}^{p} \mathrm{~d} s\right] \\
& \leq C_{N, p} t^{\frac{p}{2}-2} \int_{0}^{t} \int_{D} \mathbb{E}\left\|D \xi_{N}^{k}(s, y)\right\|_{\mathcal{H}_{T}}^{p} \mathrm{~d} y \mathrm{~d} s,
\end{aligned}
$$

provided $p>4$.

For the last term $I_{3}$, using as above Minkowski's and Hölder's inequalities, we have

$$
\begin{aligned}
\mathbb{E}\left\|I_{3}\right\|_{\mathcal{H}_{T}}^{p}=\mathbb{E} & \left.\| \int_{r}^{t} \int_{D} \nabla_{y} g(t-s, x, y) \cdot \tilde{q}_{N}\left(\xi_{N}^{k}\right)(s, \cdot)\right)(y) \\
& p\left\|\xi_{N}^{k}(s, \cdot)\right\|_{L^{p}}^{1-p}\left(\int_{D}\left|\xi_{N}^{k}(s, \beta)\right|^{p-2} \xi_{N}^{k}(s, \beta) D \xi_{N}^{k}(s, \beta) \mathrm{d} \beta\right) \mathrm{d} y \mathrm{~d} s \|_{\mathcal{H}_{T}}^{p} \\
\leq & \mathbb{E}\left[\int_{0}^{t} \int_{D} \mid \nabla_{y} g(t-s, x, y) \cdot \tilde{q}_{N}\left(\xi_{N}^{k}\right)(s, \cdot)\right)(y) \mid \\
\leq & \mathbb{E}\left[\int_{N}^{k} p\left\|\nabla_{y} g(t-)\right\|_{L^{p}}^{1-p}\left(\int_{D}\left|\xi_{N}^{k}(s, \beta)\right|^{p-1}\left\|D \xi_{N}^{k}(s, \beta)\right\|_{\mathcal{H}_{T}} \mathrm{~d} \beta\right) \mathrm{d} y \mathrm{~d} s\right]^{p}
\end{aligned}
$$

(2.8) and (4.9) imply that

$$
\begin{aligned}
\left\|\nabla_{y} g(t-s, x, \cdot) \cdot \tilde{q}_{N}\left(\xi_{N}^{k}(s, \cdot)\right)\right\|_{L^{1}} & \leq\left\|\nabla_{y} g(t-s, x, \cdot)\right\|_{L^{\frac{p}{p-1}}}\left\|\tilde{q}_{N}\left(\xi_{N}^{k}(s, \cdot)\right)\right\|_{L^{p}} \\
& \leq C_{p}(N+1)^{2}(t-s)^{-\frac{p+2}{2 p}} \quad \text { provided } p>4 .
\end{aligned}
$$

Thanks to Hölder's inequality,

$$
\begin{aligned}
& \mathbb{E}\left\|I_{3}\right\|_{\mathcal{H}_{T}}^{p} \leq C_{N, p} \mathbb{E} {\left[\int_{0}^{t}(t-s)^{-\frac{p+2}{2 p}}\left\|D \xi_{N}^{k}(s, \cdot)\right\|_{L^{p}\left(D ; \mathcal{H}_{T}\right)} \mathrm{d} s\right]^{p} } \\
& \leq C_{N, p} t^{\frac{p}{2}-2} \int_{0}^{t} \int_{D} \mathbb{E}\left\|D \xi_{N}^{k}(s, y)\right\|_{\mathcal{H}_{T}}^{p} \mathrm{~d} y \mathrm{~d} s
\end{aligned}
$$


provided $p>4$.

From the above estimates, if $p>4$, we infer

$$
\mathbb{E}\left\|D \xi_{N}^{k+1}(t, x)\right\|_{\mathcal{H}_{T}}^{p} \leq C_{p}+C_{N, T, p} \int_{0}^{t} \int_{D} \mathbb{E}\left\|D \xi_{N}^{k}(s, y)\right\|_{\mathcal{H}_{T}}^{p} \mathrm{~d} y \mathrm{~d} s .
$$

This proves that if $\xi_{N}^{k}(t, x) \in \mathbb{D}^{1, p}$, then $\xi_{N}^{k+1}(t, x) \in \mathbb{D}^{1, p}$. Moreover, iterating inequality (5.17) (which holds for every $(t, x) \in[0, T] \times D)$ and proceeding as in the proof of Theorem [14, we obtain (5.9). What remains to prove is equality (5.8); but this is obtained by applying the operator $D$ to both members of equation (4.13).

5.2. Nondegeneracy condition. Now we check condition (5.1) for the solution $\xi_{N}$ to the truncated equation. Let $t \in[0, T]$ and $x \in D$. We aim at proving that

$$
\left\|D \xi_{N}(t, x)\right\|_{\mathcal{H}_{T}}^{2}>0 \quad \mathbb{P}-\text { a.s. }
$$

The following lemma is an improvement of Theorem 15 and it is needed in order to prove Theorem 17. We need to consider a time interval smaller than $[0, T]$ and consider the $\mathcal{H}_{T}$-norm of $\xi_{N}(t, x)$ on $(t-\varepsilon, t)$ for some $\varepsilon>0$ small enough. For every $\varphi \in \mathcal{H}_{T}$ we define the norm

$$
\|\varphi\|_{\mathcal{H}_{(t-\varepsilon, t)}}:=\left\|\mathbf{1}_{(t-\varepsilon, t)}(\cdot) \varphi\right\|_{\mathcal{H}_{T}} .
$$

It is straightforward to get

$$
\|\varphi\|_{\mathcal{H}_{T}} \geq\|\varphi\|_{\mathcal{H}_{(t-\varepsilon, t)}} .
$$

Lemma 16. Let $N \geq 1, b>1$ in (2.23) and $p>4$. If $\xi_{0}$ is a continuous function on $D$, then there exists a constant $C_{N, p, Q, T}$ such that for every $0<\varepsilon<t$

$$
\sup _{\sigma \in[t-\varepsilon, t]} \sup _{x \in D} \mathbb{E}\left\|D \xi_{N}(\sigma, x)\right\|_{\mathcal{H}(t-\varepsilon, t)}^{p} \leq C_{N, p, Q, T} \varepsilon^{\frac{p}{2}} .
$$

Proof. For $t-\varepsilon \leq \sigma \leq t$, set $\eta_{N}^{\varepsilon}(\sigma, x)=\mathbb{E}\left\|D \xi_{N}(\sigma, x)\right\|_{\mathcal{H}_{(t-\varepsilon, \sigma)}}^{p}$. According to (5.8),

$$
\eta_{N}^{\varepsilon}(\sigma, x) \leq C_{p}\left(\left\|g(\sigma-\cdot, x, \cdot) \mathbf{1}_{[0, \sigma]}(\cdot)\right\|_{\mathcal{H}_{(t-\varepsilon, \sigma)}}^{p}+\sum_{i=1}^{3} \mathbb{E}\left\|I_{i}\right\|_{\mathcal{H}_{(t-\varepsilon, \sigma)}}^{p}\right)
$$

where the terms $I_{i}, i=1,2,3$, are defined in (5.11)-(5.13). By (2.24) and the change of variables $s=r-\sigma+\varepsilon$, we get

$$
\begin{gathered}
\int_{t-\varepsilon}^{\sigma}\|g(\sigma-r, x, \cdot)\|_{L_{Q}^{2}}^{2} \mathrm{~d} r \leq \sum_{k \in \mathbb{Z}_{0}^{2}}|k|^{-2 b} \int_{0}^{\varepsilon} e^{-2|k|^{2}(\varepsilon-s)}\left|e_{k}(x)\right|^{2} \mathrm{~d} s \\
=\frac{1}{(2 \pi)^{2}} \sum_{k \in \mathbb{Z}_{0}^{2}} \frac{|k|^{-2 b-2}}{2}\left(1-e^{-2|k|^{2} \varepsilon}\right) \leq \frac{1}{(2 \pi)^{2}} \sum_{k \in \mathbb{Z}_{0}^{2}} \frac{|k|^{-2 b-2}}{2}\left(2|k|^{2} \varepsilon\right) \\
=\frac{\varepsilon}{(2 \pi)^{2}} \sum_{k \in \mathbb{Z}_{0}^{2}}|k|^{-2 b}=\frac{\varepsilon}{(2 \pi)^{2}} \operatorname{Tr} Q
\end{gathered}
$$


which is finite provided $b>1$. So

$$
\begin{aligned}
\mathbb{E}\left\|g(\sigma-\cdot, x, \cdot) \mathbf{1}_{[0, \sigma]}(\cdot)\right\|_{\mathcal{H}_{(t-\varepsilon, \sigma)}}^{p} & =\left(\int_{t-\varepsilon}^{\sigma}\|g(\sigma-r, x, \cdot)\|_{L_{Q}^{2}}^{2} \mathrm{~d} r\right)^{\frac{p}{2}} \\
& \leq \frac{(\operatorname{Tr} Q)^{\frac{p}{2}} \varepsilon^{\frac{p}{2}}}{(2 \pi)^{p}}=C_{p, Q} \varepsilon^{\frac{p}{2}}
\end{aligned}
$$

Minkowski's and Hölder's inequalities and (2.17) imply that

$$
\begin{aligned}
& \mathbb{E}\left\|I_{1}\right\|_{\mathcal{H}(t-\varepsilon, \sigma)}^{p}=\mathbb{E}\left[\int_{t-\varepsilon}^{\sigma} \| \int_{r}^{\sigma} \int_{D} \nabla_{y} g(t-s, x, y) \cdot v_{N}(s, y)\right. \\
& \left.\Theta_{N}\left(\left\|\xi_{N}(s, \cdot)\right\|_{L^{p}}\right) D_{r,} \xi_{N}(s, y) \mathrm{d} y \mathrm{~d} s \|_{L_{Q}^{2}}^{2} \mathrm{~d} r\right]^{\frac{p}{2}} \\
& =\mathbb{E}\left[\int_{t-\varepsilon}^{\sigma} \| \int_{t-\varepsilon}^{\sigma} \int_{D} \nabla_{y} g(t-s, x, y) \cdot v_{N}(s, y)\right. \\
& \left.\Theta_{N}\left(\left\|\xi_{N}(s, \cdot)\right\|_{L^{p}}\right) D_{r,}, \xi_{N}(s, y) \mathrm{d} y \mathrm{~d} s \|_{L_{Q}^{2}}^{2} \mathrm{~d} r\right]^{\frac{p}{2}} \\
& \leq \mathbb{E}\left[\int_{t-\varepsilon}^{\sigma} \int_{D}\left|\nabla_{y} g(t-s, x, y) \cdot v_{N}(s, y)\right|\right. \\
& \left.\left|\Theta_{N}\left(\left\|\xi_{N}(s, \cdot)\right\|_{L^{p}}\right)\right|\left\|D \xi_{N}(s, y)\right\|_{\mathcal{H}_{(t-\varepsilon, \sigma)}} \mathrm{d} y \mathrm{~d} s\right]^{p} \\
& \leq C_{N}\left(\int_{0}^{T} \int_{D}\left|\nabla_{y} g(t-s, x, y)\right|^{\frac{p}{p-1}} \mathrm{~d} y \mathrm{~d} s\right)^{p-1} \\
& \int_{t-\varepsilon}^{\sigma} \int_{D} \mathbb{E}\left\|D \xi_{N}(s, y)\right\|_{\mathcal{H}_{(t-\varepsilon, s)}}^{p} \mathrm{~d} y \mathrm{~d} s \\
& \leq C_{N} T^{\frac{p}{2}-2} \int_{t-\varepsilon}^{\sigma} \sup _{y \in D} \mathbb{E}\left\|D \xi_{N}(s, y)\right\|_{\mathcal{H}_{(t-\varepsilon, s)}}^{p} \mathrm{~d} s \quad \text { by (2.9) } \text { if } p>4 \text {. }
\end{aligned}
$$


As regards the term $I_{2}$, proceeding in a similar way, by means of Fubini Theorem, Hölder's and Minkowski's inequalities we get

$$
\begin{aligned}
& \mathbb{E}\left\|I_{2}\right\|_{\mathcal{H}_{(t-\varepsilon, \sigma)}^{p}}^{p} \\
& =\mathbb{E}\left[\int_{t-\varepsilon}^{\sigma} \| \int_{t-\varepsilon}^{\sigma} \int_{D}\left(\nabla_{y} g(t-s, x, y) \cdot \int_{D} k(y-\alpha) D_{r,}, \xi_{N}(s, \alpha) \mathrm{d} \alpha\right)\right. \\
& \left.\Theta_{N}\left(\left\|\xi_{N}(s, \cdot)\right\|_{L^{p}}\right) \xi_{N}(s, y) \mathrm{d} y \mathrm{~d} s \|_{L_{Q}^{2}}^{2} \mathrm{~d} r\right]^{\frac{p}{2}} \\
& \leq \mathbb{E}\left[\int_{t-\varepsilon}^{\sigma} \int_{D}\left|\int_{D} \nabla_{y} g(t-s, x, y) \cdot k(y-\alpha) \xi_{N}(s, y) \Theta_{N}\left(\left\|\xi_{N}(s, \cdot)\right\|_{L^{p}}\right) \mathrm{d} y\right|\right. \\
& \left.\left\|D \xi_{N}(s, \alpha)\right\|_{\mathcal{H}_{(t-\varepsilon, \sigma)}} \mathrm{d} \alpha \mathrm{d} s\right]^{p} \\
& \leq C_{N} \mathbb{E}\left[\int_{t-\varepsilon}^{\sigma} \int_{D}\left\|\nabla_{y} g(t-s, x, \cdot) \cdot k(\cdot-\alpha)\right\|_{L^{\frac{p}{p-1}}}\left\|D \xi_{N}(s, \alpha)\right\|_{\mathcal{H}_{(t-\varepsilon, \sigma)}} \mathrm{d} \alpha \mathrm{d} s\right]^{p} \\
& \leq C_{N}\left(\int_{0}^{T}(t-s)^{\frac{p+2}{2(1-p)}} \mathrm{d} s\right)^{p-1} \\
& \int_{t-\varepsilon}^{\sigma} \int_{D} \mathbb{E}\left\|D \xi_{N}(s, y)\right\|_{\mathcal{H}_{(t-\varepsilon, s)}^{p}}^{p} \mathrm{~d} y \mathrm{~d} s \quad \text { by }(\underline{5.15)} \text { if } p>4 \\
& \leq C_{N} T^{\frac{p}{2}-2} \int_{t-\varepsilon}^{\sigma} \sup _{y \in D} \mathbb{E}\left\|D \xi_{N}(s, y)\right\|_{\mathcal{H}_{(t-\varepsilon, s)}}^{p} \mathrm{~d} s .
\end{aligned}
$$

For the last term $I_{3}$, Minkowski's any Hölder's inequalities imply that

$$
\begin{aligned}
& \mathbb{E}\left\|I_{3}\right\|_{\mathcal{H}_{(t-\varepsilon, \sigma)}}^{p}=\mathbb{E}\left[\int_{t-\varepsilon}^{\sigma}\left\|p \int_{t-\varepsilon}^{\sigma} \int_{D} \nabla_{y} g(t-s, x, y) \cdot \tilde{q}_{N}\left(\xi_{N}(s, \cdot)\right)(y)\right\| \xi_{N}(s, \cdot) \|_{L^{p}}^{1-p}\right. \\
& \left.\left(\int_{D}\left|\xi_{N}(s, \beta)\right|^{p-2} \xi_{N}(s, \beta) D_{r, \cdot} \xi_{N}(s, \beta) \mathrm{d} \beta\right) \mathrm{d} y \mathrm{~d} s \|_{L_{Q}^{2}}^{2} \mathrm{~d} r\right]^{\frac{p}{2}} \\
& \leq \mathbb{E}\left[\int_{t-\varepsilon}^{\sigma} \int_{D} p\left|\nabla_{y} g(t-s, x, y) \cdot \tilde{q}_{N}\left(\xi_{N}(s, \cdot)\right)(y)\right|\left\|\xi_{N}(s, \cdot)\right\|_{L^{p}}^{1-p}\right. \\
& \left.\leq C_{N, p} \mathbb{E}\left[\int_{t-\varepsilon}^{\sigma} p\left\|\nabla_{y} g(t-s, x, \cdot)\right\|_{L^{\frac{p}{p-1}}}\left\|D \xi_{N}(s, \cdot)\right\|_{L^{p}(D ; \mathcal{H}}(t-\varepsilon, \sigma)\right)\right]^{p} \text { by (44.9) } \\
& \left.\left.\leq C_{N, p}\left(\int_{0}^{T} \int_{D}\left|\nabla_{y} g(t-s, x, y)\right|^{\frac{p}{p-1}} \mathrm{~d} y \mathrm{~d} s\right)^{p-1} \mathrm{~d} y \mathrm{~d} s\right]^{p-1}\left\|D \xi_{N}(s, \beta)\right\|_{\mathcal{H}_{(t-\varepsilon, \sigma)}} \mathrm{d} \beta\right) \\
& \int_{t-\varepsilon}^{\sigma} \int_{D} \mathbb{E}\left\|D \xi_{N}(s, y)\right\|_{\mathcal{H}_{(t-\varepsilon, s)}}^{p} \mathrm{~d} y \mathrm{~d} s \\
& \leq C_{N, p} T^{\frac{p}{2}-2} \int_{t-\varepsilon}^{\sigma} \sup _{y \in D} \mathbb{E}\left\|D \xi_{N}(s, y)\right\|_{\mathcal{H}_{(t-\varepsilon, s)}}^{p} \mathrm{~d} s \quad \text { by (2.9) if } p>4 .
\end{aligned}
$$


Collecting the above estimates we get

$$
\sup _{x \in D} \eta_{N}^{\varepsilon}(\sigma, x) \leq C_{p, Q} \varepsilon^{\frac{p}{2}}+C_{N, p, T} \int_{t-\varepsilon}^{\sigma} \sup _{y \in D} \eta_{N}^{\varepsilon}(s, y) \mathrm{d} s \quad \text { for every } \sigma \in[t-\varepsilon, t] .
$$

By the Gronwall's lemma it follows

$$
\sup _{x \in D} \eta_{N}^{\varepsilon}(\sigma, x) \leq C_{p, Q} \varepsilon^{\frac{p}{2}} e^{C_{N, p, T}(\sigma-t+\varepsilon)} \leq C_{p, Q} \varepsilon^{\frac{p}{2}} e^{C_{N, p, T} T} \quad \text { for every } \sigma \in[t-\varepsilon, t] .
$$

Since for $\sigma \in[t-\varepsilon, t],\left\|D \xi_{N}(\sigma, x)\right\|_{\mathcal{H}_{(t-\varepsilon, \sigma)}}^{p}=\left\|D \xi_{N}(\sigma, x)\right\|_{\mathcal{H}_{(t-\varepsilon, t)}}^{p}$ we finally get

$$
\sup _{\sigma \in[t-\varepsilon, t]} \sup _{x \in D} \mathbb{E}\left\|D \xi_{N}(\sigma, x)\right\|_{\mathcal{H}_{(t-\varepsilon, t)}^{p}}^{p} \leq C_{N, p, Q, T} \varepsilon^{\frac{p}{2}} .
$$

Theorem 17. Suppose $b>1$ in (2.23) and assume that $\xi_{0}$ is a continuous function on D. Then, for every $t \in[0, T]$ and $x \in D$, the image law of the random variable $\xi_{N}(t, x)$ is absolutely continuous with respect to the Lebesgue measure on $\mathbb{R}$.

Proof. In order to prove that $\left\|D \xi_{N}(t, x)\right\|_{\mathcal{H}_{T}}^{2}>0 \quad \mathbb{P}-$ a.s. we will show that

$$
\mathbb{P}\left(\left\|D \xi_{N}(t, x)\right\|_{\mathcal{H}_{T}}^{2}=0\right)=0,
$$

or, better, that

$$
\mathbb{P}\left(\left\|D \xi_{N}(t, x)\right\|_{\mathcal{H}_{T}}^{2}<\delta\right) \rightarrow 0 \quad \text { as } \delta \rightarrow 0 .
$$

Let us fix $\varepsilon>0$ sufficiently small, according to (5.8), by means of the inequality $(a+b)^{2} \geq \frac{1}{2} a^{2}-b^{2}$, we get

$$
\begin{aligned}
\left\|D \xi_{N}(t, x)\right\|_{\mathcal{H}_{T}}^{2} & =\int_{0}^{T}\left\|D_{r, \cdot} \xi_{N}(t, x)\right\|_{L_{Q}^{2}}^{2} \mathrm{~d} r \geq \int_{t-\varepsilon}^{t}\left\|D_{r,}, \xi_{N}(t, x)\right\|_{L_{Q}^{2}}^{2} \mathrm{~d} r \\
& =\int_{t-\varepsilon}^{t}\left\|g(t-r, x, \cdot) \mathbf{1}_{[0, t]}(r)+\sum_{i=1}^{3} I_{i}(r, \cdot)\right\|_{L_{Q}^{2}}^{2} \mathrm{~d} r \\
& \geq \frac{1}{2} \int_{t-\varepsilon}^{t}\|g(t-r, x, \cdot)\|_{L_{Q}^{2}}^{2} \mathrm{~d} r-\int_{t-\varepsilon}^{t}\left\|\sum_{i=1}^{3} I_{i}(r, \cdot)\right\|_{L_{Q}^{2}}^{2} \mathrm{~d} r,
\end{aligned}
$$

where the terms $I_{i}$ are defined in (5.11)-(5.13). Let us set for simplicity

$$
I(t, x, \varepsilon)=\int_{t-\varepsilon}^{t}\left\|\sum_{i=1}^{3} I_{i}(r, \cdot)\right\|_{L_{Q}^{2}}^{2} \mathrm{~d} r, \quad A(x, \varepsilon)=\int_{t-\varepsilon}^{t}\|g(t-r, x, \cdot)\|_{L_{Q}^{2}}^{2} \mathrm{~d} r .
$$

By means of Chebyschev's inequality, for $\delta>0$ sufficiently small, we have

$$
\mathbb{P}\left(\left\|D \xi_{N}(t, x)\right\|_{\mathcal{H}_{T}}^{2}<\delta\right) \leq \mathbb{P}\left(I(t, x, \varepsilon) \geq \frac{1}{2} A(x, \varepsilon)-\delta\right) \leq \frac{\mathbb{E}|I(t, x, \varepsilon)|^{\frac{p}{2}}}{\left(\frac{1}{2} A(x, \varepsilon)-\delta\right)^{\frac{p}{2}}}
$$

Let us find an upper estimate for $\mathbb{E}|I(t, x, \varepsilon)|^{\frac{p}{2}} \leq C_{p} \sum_{i=1}^{3} \mathbb{E}\left|\int_{t-\varepsilon}^{t}\left\|I_{i}(r, \cdot)\right\|_{L_{Q}^{2}}^{2} \mathrm{~d} r\right|^{\frac{p}{2}}$. 
Minkowski's and Hölder's inequalities and (2.17) imply that

$$
\begin{aligned}
& \mathbb{E}\left|\int_{t-\varepsilon}^{t}\left\|I_{1}(r, \cdot)\right\|_{L_{Q}^{2}}^{2} \mathrm{~d} r\right|^{\frac{p}{2}} \\
& =\mathbb{E}\left[\int_{t-\varepsilon}^{t} \| \int_{t-\varepsilon}^{t} \int_{D} \nabla_{y} g(t-s, x, y) \cdot v_{N}(s, y)\right. \\
& \left.\quad \Theta_{N}\left(\left\|\xi_{N}(s, \cdot)\right\|_{L^{p}}\right) D_{r,} \xi_{N}(s, y) \mathrm{d} y \mathrm{~d} s \|_{L_{Q}^{2}}^{2} \mathrm{~d} r\right]^{\frac{p}{2}} \\
& \leq C_{N}\left(\int_{t-\varepsilon}^{t} \int_{D}\left|\nabla_{y} g(t-s, x, y)\right|^{\frac{p}{p-1}} \mathrm{~d} y \mathrm{~d} s\right)^{p-1} \int_{t-\varepsilon}^{t} \int_{D} \mathbb{E}\left\|D \xi_{N}(s, y)\right\|_{\mathcal{H}_{(t-\varepsilon, t)}^{p}}^{p} \mathrm{~d} y \mathrm{~d} s
\end{aligned}
$$

Using Lemma 16 with $t-\varepsilon \leq s \leq t$ and (2.9), provided $p>4$, we deduce that

$$
\mathbb{E}\left|\int_{t-\varepsilon}^{t}\left\|I_{1}(r, \cdot)\right\|_{L_{Q}^{2}}^{2} \mathrm{~d} r\right|^{\frac{p}{2}} \leq C_{N, p, Q, T} \varepsilon^{\frac{p}{2}-2} \varepsilon^{\frac{p}{2}}=C_{N, p, Q, T} \varepsilon^{p-2} .
$$

For the term $I_{2}$, by means of Fubini Theorem, Hölder's and Minkowski's inequalities and by (5.15), provided $p>4$, we get

$$
\begin{aligned}
& \mathbb{E}\left|\int_{t-\varepsilon}^{t}\left\|I_{2}(r, \cdot)\right\|_{L_{Q}^{2}}^{2} \mathrm{~d} r\right|^{\frac{p}{2}} \\
& =\mathbb{E}\left[\int_{t-\varepsilon}^{t} \| \int_{t-\varepsilon}^{t} \int_{D}\left(\nabla_{y} g(t-s, x, y) \cdot \int_{D} k(y-\alpha) D_{r, \cdot} \xi_{N}(s, \alpha) \mathrm{d} \alpha\right)\right. \\
& \left.\quad \Theta_{N}\left(\left\|\xi_{N}(s, \cdot)\right\|_{L^{p}}\right) \xi_{N}(s, y) \mathrm{d} y \mathrm{~d} s \|_{L_{Q}^{2}}^{2} \mathrm{~d} r\right]^{\frac{p}{2}} \\
& \leq C_{N}\left(\int_{t-\varepsilon}^{t}(t-s)^{\frac{p+2}{2(1-p)}} \mathrm{d} s\right)^{p-1} \int_{t-\varepsilon}^{t} \int_{D} \mathbb{E}\left\|D \xi_{N}(s, y)\right\|_{\mathcal{H}_{(t-\varepsilon, t)}} \mathrm{d} y \mathrm{~d} s \\
& \leq C_{N} \varepsilon^{\frac{p}{2}-2} C_{N, p, Q, T} \varepsilon^{p}=C_{N, p, Q, T} \varepsilon^{p-2} \text { by Lemma 16. }
\end{aligned}
$$

As regards the last term $I_{3}$, Minkowski's and Hölder's inequalities and (4.9) imply that

$$
\begin{aligned}
& \mathbb{E}\left|\int_{t-\varepsilon}^{t}\left\|I_{3}(r, \cdot)\right\|_{L_{Q}^{2}}^{2} \mathrm{~d} r\right|^{\frac{p}{2}} \\
& =\mathbb{E}\left[\int_{t-\varepsilon}^{t}\left\|p \int_{t-\varepsilon}^{t} \int_{D} \nabla_{y} g(t-s, x, y) \cdot \tilde{q}_{N}\left(\xi_{N}(s, \cdot)\right)(y)\right\| \xi_{N}(s, \cdot) \|_{L^{p}}^{1-p}\right. \\
& \left.\quad\left(\int_{D}\left|\xi_{N}(s, \beta)\right|^{p-2} \xi_{N}(s, \beta) D_{r,} \xi_{N}(s, \beta) \mathrm{d} \beta\right) \mathrm{d} y \mathrm{~d} s \|_{L_{Q}^{2}}^{2} \mathrm{~d} r\right]^{\frac{p}{2}} \\
& \leq C_{N, p}\left(\int_{t-\varepsilon}^{t} \int_{D}\left|\nabla_{y} g(t-s, x, y)\right|^{\frac{p}{p-1}} \mathrm{~d} y \mathrm{~d} s\right)^{p-1} \\
& \quad \int_{t-\varepsilon}^{t} \int_{D} \mathbb{E}\left\|D \xi_{N}(s, y)\right\|_{\mathcal{H}_{(t-\varepsilon, t)}^{p}}^{p} \mathrm{~d} y \mathrm{~d} s \\
& \leq C_{N, p} \varepsilon^{\frac{p}{2}-2} C_{N, p, Q, T} \varepsilon^{p}=C_{N, p, Q, T} \varepsilon^{p-2} \quad \text { by Lemma [16] and (2.9) if } p>4 .
\end{aligned}
$$


In conclusion, collecting all the above estimates, we get

$$
\mathbb{E}|I(t, x, \varepsilon)|^{\frac{p}{2}} \leq C_{N, p, Q, T} \varepsilon^{p-2},
$$

provided $p>4$. We now need to find a lower estimate for $A(x, \varepsilon)$. Proceeding as in (2.24) we have

$$
\int_{t-\varepsilon}^{t}\|g(t-r, x, \cdot)\|_{L_{Q}^{2}}^{2} \mathrm{~d} r=\sum_{k \in \mathbb{Z}_{0}^{2}}|k|^{-2 b}\left|e_{k}(x)\right|^{2} \frac{1}{2|k|^{2}}\left(1-e^{-2|k|^{2} \varepsilon}\right) .
$$

The inequality

$$
1-e^{-2|k|^{2} \varepsilon} \geq \frac{2 \varepsilon|k|^{2}}{1+2 \varepsilon|k|^{2}} \geq \frac{2 \varepsilon|k|^{2}}{1+2 T|k|^{2}}
$$

implies that

$$
\int_{t-\varepsilon}^{t}\|g(t-r, x, \cdot)\|_{L_{Q}^{2}}^{2} \mathrm{~d} r \geq \frac{\varepsilon}{(2 \pi)^{2}} \sum_{k \in \mathbb{Z}_{0}^{2}} \frac{|k|^{-2 b}}{1+2 T|k|^{2}}
$$

and the above series is well defined and can be bounded from below by any of its summand, such as the one corresponding to $k=(0,1) \in \mathbb{Z}_{0}^{2}$ :

$$
\int_{t-\varepsilon}^{t}\|g(t-r, x, \cdot)\|_{L_{Q}^{2}}^{2} \mathrm{~d} r \geq \frac{\varepsilon}{(2 \pi)^{2}(1+2 T)}=C_{T} \varepsilon .
$$

Using estimates (5.23) and (5.24) and substituting into (5.22) we get

$$
\mathbb{P}\left(\left\|D \xi_{N}(t, x)\right\|_{\mathcal{H}_{T}}^{2}<\delta\right) \leq\left(\frac{C_{T}}{2} \varepsilon-\delta\right)^{-\frac{p}{2}} C_{N, p, Q, T} \varepsilon^{p-2} .
$$

Thus, if we choose $\varepsilon=\varepsilon(\delta, T)$ sufficiently small in such a way that $\frac{C_{T}}{2} \varepsilon=2 \delta$ we get

$$
\mathbb{P}\left(\left\|D \xi_{N}(t, x)\right\|_{\mathcal{H}_{T}}^{2}<\delta\right) \leq C_{N, T, Q, p} \delta^{-\frac{p}{2}} \delta^{p-2}=C_{N, T, Q, p} \delta^{\frac{p}{2}-2} \rightarrow 0 \quad \text { for } \delta \rightarrow 0,
$$

since $p>4$.

5.3. Existence of the density. Now we are ready to prove the main result, Theorem 2 .

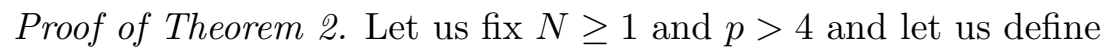

$$
\Omega_{N}:=\left\{\omega \in \Omega: \sup _{t \in[0, T]}\|\xi(t, \cdot, \omega)\|_{L^{p}(D)} \leq N\right\} .
$$

Then we have $\xi(t, x) \equiv \xi_{N}(t, x)$ on $\Omega_{N}$ for every $t \in[0, T]$ and $x \in D$ and $\lim _{N \rightarrow+\infty} \mathbb{P}\left(\Omega_{N}=\Omega\right)=1$. In fact we can write

$$
\Omega_{N}=\left\{\sigma_{N}=T\right\},
$$

where $\sigma_{N}$ is the stopping time defined in (4.18). So we have that, for $N \rightarrow \infty, \sup _{N \geq 1} \sigma_{N}=T \mathbb{P}$-a.s. i.e. $\Omega_{N} \uparrow \Omega \mathbb{P}$-a.s.

It follows then that, for every $(t, x) \in[0, T] \times D)$, the sequence $\left(\Omega_{N}, \xi_{N}(t, x)\right)$ localizes $\xi(t, x)$ in $\mathbb{D}^{1, p}$. The result follows by Theorem 17, in fact it suffices to show property (5.1) on the set $\left\{t<\sigma_{N}\right\}$ for every $N \geq 1$, namely to show (5.18). 


\section{Appendix A. Proof of Theorem 4}

For the estimate of the heat kernel and its gradient we use the explicit expression given by (2.7). We factorize the two-dimensional kernel into the one dimensional components. We then proceed following the idea of [21, Lemma 2.1].

$$
\begin{aligned}
g(t, x, y) & =\frac{1}{4 \pi t} \sum_{k \in \mathbb{Z}^{2}} e^{-\frac{|x-y+2 \pi k|^{2}}{4 t}} \\
& =\left(\frac{1}{\sqrt{4 \pi t}} \sum_{k_{1} \in \mathbb{Z}} e^{\frac{-\left|x_{1}-y_{1}+2 \pi k_{1}\right|^{2}}{4 t}}\right)\left(\frac{1}{\sqrt{4 \pi t}} \sum_{k_{2} \in \mathbb{Z}} e^{\frac{-\left|x_{2}-y_{2}+2 \pi k_{2}\right|^{2}}{4 t}}\right) .
\end{aligned}
$$

Let us set, for $i=1,2$

$$
g_{i}\left(t, x_{i}, y_{i}\right)=\frac{1}{\sqrt{4 \pi t}} \sum_{k_{i} \in \mathbb{Z}} e^{\frac{-\left|x_{i}-y_{i}+2 \pi k_{i}\right|^{2}}{4 t}}
$$

then

$$
g(t, x, y)=g_{1}\left(t, x_{1}, y_{1}\right) g_{2}\left(t, x_{2}, y_{2}\right)
$$

For the one-dimensional heat kernel the following decomposition holds:

$$
g_{i}\left(t, x_{i}, y_{i}\right)=H_{i}^{1}\left(t, x_{i}, y_{i}\right)+H_{i}^{2}\left(t, x_{i}, y_{i}\right)+H_{i}^{3}\left(t, x_{i}, y_{i}\right)+\bar{g}_{i}\left(t, x_{i}, y_{i}\right)
$$

where

$$
\begin{gathered}
H_{i}^{1}\left(t, x_{i}, y_{i}\right)=\frac{1}{\sqrt{4 \pi t}} e^{\frac{-\left|x_{i}-y_{i}\right|^{2}}{4 t}}, \quad H_{i}^{2}\left(t, x_{i}, y_{i}\right)=\frac{1}{\sqrt{4 \pi t}} e^{\frac{-\left|x_{i}-y_{i}+2 \pi\right|^{2}}{4 t}} \\
H_{i}^{3}\left(t, x_{i}, y_{i}\right)=\frac{1}{\sqrt{4 \pi t}} e^{\frac{-\left|x_{i}-y_{i}-2 \pi\right|^{2}}{4 t}}
\end{gathered}
$$

and

$$
\left(t, x_{i}, y_{i}\right) \rightarrow \bar{g}_{i}\left(t, x_{i}, y_{i}\right) \in C^{\infty}\left([0, T] \times \mathbb{R}^{2}\right) .
$$

Then we can rewrite the two dimensional heat kernel as follows

$$
\begin{aligned}
g(t, x, y)=\left(H_{1}^{1}\left(t, x_{1}, y_{1}\right)+H_{1}^{2}\left(t, x_{1}, y_{1}\right)+H_{1}^{3}\left(t, x_{1}, y_{1}\right)+\bar{g}_{1}\left(t, x_{1}, y_{1}\right)\right) & \\
& \left(H_{2}^{1}\left(t, x_{2}, y_{2}\right)+H_{2}^{2}\left(t, x_{2}, y_{2}\right)+H_{2}^{3}\left(t, x_{2}, y_{2}\right)+\bar{g}_{2}\left(t, x_{2}, y_{2}\right)\right) .
\end{aligned}
$$

We are interested in estimating the heat kernel and its gradient, more precisely in estimates of the following type:

$$
\int_{0}^{t} \int_{D}|g(s, x, y)|^{\beta} \mathrm{d} y \mathrm{~d} s, \quad \quad \int_{0}^{t} \int_{D}\left|\nabla_{y} g(s, x, y)\right|^{\beta} \mathrm{d} y \mathrm{~d} s,
$$

for $t>0$ and a suitable $\beta>0$. 
Remark 3. Let us notice that the terms of the form $H_{1}^{k} \bar{g}_{2}$ and $H_{2}^{k} \bar{g}_{1}$ with $k=1,2,3$ do not give any problems. In fact let us consider for example the case $H_{1}^{1} \bar{g}_{2}$ (the others are similar). We have

$$
\begin{aligned}
& \left|\nabla_{y}\left(H_{1}^{1} \bar{g}_{2}\right)\right|^{\beta}=\left(\left|\nabla_{y}\left(H_{1}^{1} \bar{g}_{2}\right)\right|^{2}\right)^{\frac{\beta}{2}} \\
& \leq C_{\beta}\left(\frac{\left(2\left|x_{1}-y_{1}\right|\right)^{\beta}}{\pi^{\frac{\beta}{2}}(4 t)^{\frac{3 \beta}{2}}} e^{-\frac{\beta\left|x_{1}-y_{1}\right|^{2}}{4 t}}\left|\bar{g}_{2}\left(t, x_{2}, y_{2}\right)\right|^{\beta}+\frac{1}{(4 \pi t)^{\frac{\beta}{2}}} e^{-\frac{\beta\left|x_{1}-y_{1}\right|^{2}}{4 t}}\left|\frac{\partial}{\partial y_{2}} \bar{g}_{2}\left(t, x_{2}, y_{2}\right)\right|^{\beta}\right) \\
& \leq C_{\beta} \frac{\left|x_{1}-y_{1}\right|^{\beta}}{t^{\frac{3 \beta}{2}}} e^{-\frac{\beta\left|x_{1}-y_{1}\right|^{2}}{4 t}}\left|\bar{g}_{2}\left(t, x_{2}, y_{2}\right)\right|^{\beta}+\frac{C_{\beta}}{t^{\frac{\beta}{2}}} e^{-\frac{\beta\left|x_{1}-y_{1}\right|^{2}}{4 t}}\left|\frac{\partial}{\partial y_{2}} \bar{g}_{2}\left(t, x_{2}, y_{2}\right)\right|^{\beta} .
\end{aligned}
$$

Then, using the following identity

$$
\int_{\mathbb{R}}|z|^{r} e^{-\frac{z^{2}}{\sigma^{2}}} \mathrm{~d} z=C_{r} \sigma^{r+1}
$$

we get

$$
\begin{aligned}
\int_{0}^{t} \int_{D} \mid & \left.\nabla_{y}\left(H_{1}^{1} \bar{g}_{2}\right)(s, x, y)\right|^{\beta} \mathrm{d} y \mathrm{~d} s \\
\leq & C_{\beta} \int_{0}^{t} \int_{0}^{2 \pi}\left(\int_{0}^{2 \pi} \frac{\left|x_{1}-y_{1}\right|^{\beta}}{s^{\frac{3 \beta}{2}}} e^{-\frac{\beta\left|x_{1}-y_{1}\right|^{2}}{4 s}} \mathrm{~d} y_{1}\right)\left|\bar{g}_{2}\left(s, x_{2}, y_{2}\right)\right|^{\beta} \mathrm{d} y_{2} \mathrm{~d} s \\
& +C_{\beta} \int_{0}^{t} \frac{1}{s^{\frac{\beta}{2}}} \int_{0}^{2 \pi}\left(\int_{0}^{2 \pi} e^{-\frac{\beta\left|x_{1}-y_{1}\right|^{2}}{4 s}} \mathrm{~d} y_{1}\right)\left|\frac{\partial}{\partial y_{2}} \bar{g}_{2}\left(s, x_{2}, y_{2}\right)\right|^{\beta} \mathrm{d} y_{2} \mathrm{~d} s \\
\leq & C_{\beta} \int_{0}^{t} \int_{0}^{2 \pi} s^{\frac{1}{2}-\beta}\left|\bar{g}_{2}\left(s, x_{2}, y_{2}\right)\right|^{\beta} \mathrm{d} y_{2} \mathrm{~d} s \\
& +C_{\beta} \int_{0}^{t} \int_{0}^{2 \pi} s^{\frac{1-\beta}{2}}\left|\frac{\partial}{\partial y_{2}} \bar{g}_{2}\left(s, x_{2}, y_{2}\right)\right|^{\beta} \mathrm{d} y_{2} \mathrm{~d} s
\end{aligned}
$$

and we have the convergence of the integrals thanks to (A.1), when $\beta<\frac{3}{2}$.

By Remark 3 it follows that the behavior of integrals in (A.2) is determined by the corresponding integrals with $H_{1}^{k} H_{2}^{l}$ with $k, l=1,2,3$, instead of $g$. Since computations are similar we do all the required estimates only for the case $H(t, x, y):=H_{1}^{1}\left(t, x_{1}, y_{1}\right) H_{2}^{1}\left(t, x_{2}, y_{2}\right)$. We have

$$
\left|\nabla_{y} H(t, x, y)\right|^{\beta}=\frac{e^{-\frac{\beta|x-y|^{2}}{4 t}}|x-y|^{\beta}}{(8 \pi)^{\beta} t^{2 \beta}},
$$

so we recover

$$
\begin{aligned}
& \int_{D}\left|\nabla_{y} H(s, x, y)\right|^{\beta} \mathrm{d} y=\int_{D} \frac{e^{-\frac{\beta|x-y|^{2}}{4 s}}|x-y|^{\beta}}{(8 \pi)^{\beta} s^{2 \beta}} \mathrm{d} y \\
& \quad \leq C_{\beta} \int_{\mathbb{R}^{2}} \frac{e^{-\frac{\beta|z|^{2}}{4 s}}|z|^{\beta}}{s^{2 \beta}} \mathrm{d} z=C_{\beta} \int_{0}^{2 \pi} \int_{0}^{\infty} \frac{e^{-\frac{\beta \rho^{2}}{4 s}} \rho^{\beta+1}}{s^{2 \beta}} \mathrm{d} \rho \mathrm{d} \phi \\
& \leq C_{\beta} \frac{1}{s^{2 \beta}} \int_{0}^{\infty} \rho^{\beta+1} e^{-\frac{\beta \rho^{2}}{4 s}} \mathrm{~d} \rho .
\end{aligned}
$$


Using now identity (A.3) we get

$$
\int_{D}\left|\nabla_{y} H(s, x, y)\right|^{\beta} \mathrm{d} y \mathrm{~d} s \leq C_{\beta} s^{-\frac{3 \beta}{2}+1} .
$$

Calculating the time integral we obtain,

$$
\int_{0}^{t} \int_{D}\left|\nabla_{y} H(s, x, y)\right|^{\beta} \mathrm{d} y \mathrm{~d} s \leq C_{\beta} \int_{0}^{t} s^{-\frac{3 \beta}{2}+1} \mathrm{~d} s \leq C_{\beta} t^{-\frac{3 \beta}{2}+2},
$$

which converges provided $\beta<\frac{4}{3}$.

Remark 4. Notice that estimate (A.4) is uniform in $x$.

For estimates (2.10) and (2.11) we proceed in a similar way. Also in this case we do all the required estimates for $H(t, x, y):=H_{1}^{1}\left(t, x_{1}, y_{1}\right) H_{2}^{1}\left(t, x_{2}, y_{2}\right)$. By means of (A.3) we get

$$
\begin{aligned}
\int_{D}|H(s, x, y)|^{\beta} \mathrm{d} y & =\int_{D} \frac{1}{(4 \pi s)^{\beta}} e^{-\frac{\beta|x-y|^{2}}{4 s}} \mathrm{~d} y \leq C_{\beta} \int_{\mathbb{R}^{2}} \frac{1}{s^{\beta}} e^{-\frac{\beta|z|^{2}}{4 s}} \mathrm{~d} z \\
& =2 \pi C_{\beta} \int_{0}^{\infty} \frac{e^{-\frac{\beta \rho^{2}}{4 s}}}{s^{\beta}} \rho \mathrm{d} \rho \leq C_{\beta} s^{1-\beta} .
\end{aligned}
$$

Computing the time integral we obtain

$$
\int_{0}^{t} \int_{D}|H(s, x, y)|^{\beta} \mathrm{d} y \mathrm{~d} s \leq C_{\beta} \int_{0}^{t} s^{1-\beta} \mathrm{d} s \leq C_{\beta} t^{2-\beta},
$$

which converges provided $\beta<2$.

\section{REFERENCES}

[1] V. Bally and E. Pardoux. Malliavin calculus for white noise driven parabolic SPDEs. Potential Anal., 9(1):27-64, 1998.

[2] H. Bessaih and B. Ferrario. Inviscid limit of stochastic damped 2D Navier-Stokes equations. Nonlinearity, 27(1):1-15, 2014.

[3] H. Brezis. Functional analysis, Sobolev spaces and partial differential equations. Springer Science \& Business Media, 2010.

[4] Z. Brzeźniak, F. Flandoli, and M. Maurelli. Existence and uniqueness for stochastic 2D Euler flows with bounded vorticity. Arch. Ration. Mech. Anal., 221(1):107-142, 2016.

[5] C. Cardon-Weber. Cahn-Hilliard stochastic equation: existence of the solution and of its density. Bernoulli, 7(5):777816, 2001.

[6] G. Da Prato. Kolmogorov equations for stochastic PDEs. Birkhäuser Verlag, Basel, 2004.

[7] G. Da Prato. Introduction to stochastic analysis and Malliavin calculus, volume 7 of Appunti. Lecture Notes. Scuola Normale Superiore di Pisa (New Series). Edizioni della Normale, Pisa. 2008.

[8] G. Da Prato and J. Zabczyk. Stochastic equations in infinite dimensions, volume 44 of Encyclopedia of Mathematics and its Applications. Cambridge University Press, Cambridge, 1992.

[9] R. C. Dalang, D. Khoshnevisan, and E. Nualart. Hitting probabilities for systems for non-linear stochastic heat equations with multiplicative noise. Probab. Theory Related Fields, 144(3-4):371-427, 2009.

[10] R. C. Dalang and L. Quer-Sardanyons. Stochastic integrals for spde's: a comparison. Expo. Math., 29(1):67-109, 2011.

[11] R. C. Dalang and M. Sanz-Solé. Criteria for hitting probabilities with applications to systems of stochastic wave equations. Bernoulli, 16(4):1343-1368, 2010.

[12] H. Dym and H. P. McKean. Fourier Series and Integrals, volume 14 of Probability and Mathematical Statistics. Academic Press, 1972.

[13] D. Grieser. Uniform bounds for eigenfunctions of the Laplacian on manifolds with boundary. Commun. in Partial Diff. Eq., 27(7-8):1283-1299, 2002. 
[14] I. Gyöngy. Existence and uniqueness results for semilinear stochastic partial differential equations. Stochastic Process. Appl., 73(2):271-299, 1998.

[15] I. Gyöngy and D. Nualart. On the stochastic Burgers' equation in the real line. Ann. Probab., 27(2):782-802, 1999.

[16] J.-L. Lions and E. Magenes. Non-homogeneous boundary value problems and applications. Vol. I. Springer-Verlag, New York, 1972.

[17] A. J. Majda and A. L. Bertozzi. Vorticity and incompressible flow, volume 27. Cambridge University Press, 2002.

[18] C. Marchioro and M. Pulvirenti. Mathematical theory of incompressible nonviscous fluids, volume 96. Springer Science \& Business Media, 2012.

[19] C. Marinelli, E. Nualart, and L. Quer-Sardanyons. Existence and regularity of the density for solutions to semilinear dissipative parabolic SPDEs. Potential Anal., 39(3):287-311, 2013.

[20] D. Márquez-Carreras, M. Mellouk, and M. Sarrà. On stochastic partial differential equations with spatially correlated noise: smoothness of the law. Stochastic Process. Appl., 93(2):269-284, 2001.

[21] P.-L. Morien. On the density for the solution of a Burgers-type SPDE. Ann. Inst. H. Poincaré Probab. Statist., 35(4):459-482, 1999.

[22] C. Mueller and D. Nualart. Regularity of the density for the stochastic heat equation. Electron. J. Probab., 13:no. 74, 2248-2258, 2008.

[23] I. Nourdin and F. G. Viens. Density formula and concentration inequalities with Malliavin calculus. Electron. J. Probab., 14:no. 78, 2287-2309, 2009.

[24] D. Nualart. The Malliavin calculus and related topics. Probability and its Applications (New York). Springer-Verlag, Berlin, second edition, 2006.

[25] L. Quer-Sardanyons and M. Sanz-Solé. Absolute continuity of the law of the solution to the 3-dimensional stochastic wave equation. J. Funct. Anal., 206(1):1-32, 2004.

[26] E. M. Stein and G. Weiss. Introduction to Fourier analysis on Euclidean spaces. Princeton, New Jersey, 1971.

$[27]$ D. W. Stroock. A concise introduction to the theory of integration. Birkhäuser Boston Inc., Boston, MA, third edition, 1999.

[28] R. Temam. Navier-Stokes equations and nonlinear functional analysis, volume 66. Siam, 1995.

[29] J. B. Walsh. An introduction to stochastic partial differential equations. In École d'été de probabilités de Saint-Flour, XIV-1984, volume 1180 of Lecture Notes in Math., pages 265-439. Springer, Berlin, 1986.

[30] N. L. Zaidi and D. Nualart. Burgers equation driven by a space-time white noise: absolute continuity of the solution. Stochastics Stochastics Rep., 66(3-4):273-292, 1999.

Benedetta Ferrario, Margherita Zanella

Università di Pavia, Dipartimento di Matematica "F. Casorati", via Ferrata 5, 27100 Pavia, Italy

E-mail address: benedetta.ferrario@unipv.it, margherita.zanella01@ateneopv.it 